\title{
Toward Assessing and Recommending Combinations of Behaviors for Improving Health and Well-Being
}

\author{
EHIMWENMA NOSAKHARE, Massachusetts Institute of Technology \\ ROSALIND PICARD, MIT Media Lab
}

\begin{abstract}
Multiple behaviors typically work together to influence health, making it hard to understand how one behavior might compensate for another. Rich multi-modal datasets from mobile sensors and advances in machine learning are today enabling new kinds of associations to be made between combinations of behaviors objectively assessed from daily life and self-reported levels of stress, mood, and health. In this article, we present a framework to (1) map multi-modal messy data collected in the "wild" to meaningful feature representations of health-related behaviors, (2) uncover latent patterns comprising combinations of behaviors that best predict health and well-being, and (3) use these learned patterns to make evidence-based recommendations that may improve health and well-being. We show how to use supervised latent Dirichlet allocation to model the observed behaviors, and we apply variational inference to uncover the latent patterns. Implementing and evaluating the model on 5,397 days of data from a group of 244 college students, we find that these latent patterns are indeed predictive of daily self-reported levels of stressed-calm, sad-happy, and sick-healthy states. We investigate the patterns of modifiable behaviors present on different days and uncover several ways in which they relate to stress, mood, and health. This work contributes a new method using objective data analysis to help advance understanding of how combinations of modifiable human behaviors may promote human health and well-being.
\end{abstract}

CCS Concepts: • Applied computing $\rightarrow$ Consumer health; • Computing methodologies $\rightarrow$ Latent variable models;

Additional Key Words and Phrases: Mental health and well-being, latent variable modeling, supervised topic model, machine learning, recommending health behaviors, mood, health, stress

\section{ACM Reference format:}

Ehimwenma Nosakhare and Rosalind Picard. 2020. Toward Assessing and Recommending Combinations of Behaviors for Improving Health and Well-Being. ACM Trans. Comput. Healthcare 1, 1, Article 4 (February 2020), 29 pages.

https://doi.org/10.1145/3368958

\section{INTRODUCTION}

Poor mental well-being has become a world-wide problem. The World Health Organization estimates that one in four people will be affected by a mental disorder at some point in their lives. ${ }^{1}$ Depression has become the leading

\footnotetext{
$\overline{{ }^{1} \text { http://www.who.int/whr/2001/media_centre/press_release/en/. }}$

An earlier version of this article was presented at the 2019 ACM SIGKDD conference and was published in its proceedings.

This work was supported by the MIT Media Lab Consortium, NIH grant R01GM105018, Samsung Electronics, and NEC Corporation.

Authors' addresses: E. Nosakhare, Massachusetts Institute of Technology, Cambridge, MA 02139; email: ehinosa@alum.mit.edu; R. Picard, MIT Media Lab, Cambridge, MA 02139; email: picard@media.mit.edu.

Permission to make digital or hard copies of all or part of this work for personal or classroom use is granted without fee provided that copies are not made or distributed for profit or commercial advantage and that copies bear this notice and the full citation on the first page. Copyrights for components of this work owned by others than the author(s) must be honored. Abstracting with credit is permitted. To copy otherwise, or republish, to post on servers or to redistribute to lists, requires prior specific permission and/or a fee. Request permissions from permissions@acm.org.

(C) 2020 Copyright held by the owner/author(s). Publication rights licensed to ACM.

2637-8051/2020/02-ART4 \$15.00

https://doi.org/10.1145/3368958
} 
cause of disability worldwide, with an estimated 300 million people depressed. ${ }^{2}$ Further, it has been shown that not only can depression be elicited by major diseases such as heart disease, but also chronic stress and depression can independently cause physical diseases, including widespread heart disease [13] and diabetes [20]. Thus, there is an increasing need to foster research that promotes well-being and positive mental health.

Mental well-being is dynamic, and studies have shown that major contributors include daily health behaviors, as well as environmental and social factors [6]. The growing use of personal health-related mobile applications, fitness trackers, and other sensors has made it feasible to collect data to better characterize environmental and lifestyle factors driving mental health outcomes [21]. These devices are able to collect real-time information, with the average user likely to produce more than 1 million gigabytes of health-related data in his or her lifetime-the equivalent of about 300 million books. ${ }^{3}$ Although there are still limitations, the availability of these multi-modal data sources, with advances in machine learning methods, suggest that it is possible to objectively assess how our daily behaviors and activities influence mental health and well-being.

Typically, studies investigate daily life health and behavior data to find effects of a stand-alone behavior on health and well-being [14, 28, 30]. A problem is that behaviors typically work together and do not affect wellbeing in isolation, with different combinations of behaviors possibly compensating for each other, and resulting in different health outcomes. To that end, understanding how complex factors-behavioral, environmental, and social-work together could help provide insights into the groups of behaviors to target when looking to improve an individual's well-being. There is therefore a need to develop a new solution providing personalized models that are able to analyze how complex groups of behaviors contribute to the state of well-being, and provide actionable evidence-based insights to individuals who wish to improve their mental health.

In this article, we address this complex real-world problem of extracting and identifying important patterns of health behaviors given raw multi-modal data collected in the "wild." The specific group we address is college students, who provided the sensor and smartphone data we use; however, the framework we present is general. We present a new framework for uncovering patterns of modifiable human behaviors that influence the wellbeing of individuals and using these patterns to provide evidence-based recommendations to individuals seeking to improve their well-being. We posit that there are latent patterns responsible for the set of health behaviors observed in an individual on any given day, and that these patterns consequently contribute to that individual's well-being.

Our solution is threefold and employs latent Dirichlet allocation (LDA), a method commonly used in summarizing topics within documents of text. First, we develop a way to map the students' real-world messy data to meaningful feature representations of modifiable health behaviors, analogous to identifying words that can be changed within a document. Second, we use supervised LDA (sLDA) to model the observed behaviors, and we apply variational inference to uncover latent patterns that give rise to the observations (analogous to uncovering the important topics undergirding a set of documents). We then evaluate the learned latent patterns to see how well they predict three categories of student well-being-mood, stress, and physical health-as measured by daily self-reported labels. We find the sLDA patterns are indeed predictive of well-being and perform significantly better than a control model that obtains patterns in an unsupervised way. We also investigate the student behaviors present in these patterns and examine how they work together to influence the well-being of each individual student. Third, we illustrate how we can use the learned patterns to recommend feasible behavioral changes that may affect a future outcome. The knowledge achieved by this new framework provides insights into how multiple real-world behaviors might affect health; further, the results help identify candidate variables for future causality studies, both on a group level and on a personalized level.

Our multi-modal dataset comes from a prior study that measured Sleep, Networks, Affect, Performance, Stress and Health using Objective Techniques (SNAPSHOT) continuously for 30 days in college students [24, 26].

\footnotetext{
${ }^{2}$ http://www.who.int/news-room/fact-sheets/detail/depression/.

${ }^{3}$ https://www-03.ibm.com/press/uk/en/pressrelease/46609.wss/. 
Chronic stress leads to depression, and college years are usually very high stress periods for most young adults. This trend is unfortunately on the increase-in 2017, there was a $12 \%$ increase in American college students reporting more than average to tremendous stress when compared to $2013 .{ }^{4}$ The high stress period of college years provide a useful case study for demonstrating the new framework. In addition, the student behaviors are highly heterogeneous, improving the likelihood that the health findings may apply beyond this dataset.

In Section 2, we highlight current approaches of modeling human behavior in the context of well-being and the machine learning methods used. In Section 3, we briefly overview our approach and the novel contributions of this work. In Section 4, we describe the data used in this work, emphasizing a method of providing modifiable behaviors as features. In Section 5, we present our methodology for moving from messy daily data to predicting useful patterns: First, we introduce a method of extracting behaviors from complex multi-modal real-world data and binning them to ensure that they are descriptive of a participant's day. Second, we discuss how we model interpretable latent patterns of health behaviors that are predictive of health and well-being states, and we present the methods used for evaluation compared to a control. Third, we illustrate how we use these latent patterns to provide evidence-based insights useful for improving well-being. Section 6 presents results and discussion of the findings, and Section 7 closes with conclusions.

\section{RELATED WORK}

Previous work on the SNAPSHOT dataset has focused on predicting well-being as defined by self-reported stress, mood and health. Taylor et al. [29] used multi-task learning Bayesian and neural network approaches to predict future well-being, achieving binary prediction accuracies in the range of $72 \%$ to $83 \%$. Sano et al. [26] reported that wearable sensor features such as skin temperature and skin conductance were important features in the classification of high/low stress and mental health. Predicting an individual's well-being is important and can be useful for motivating behavior change (e.g., "What can I do tonight to change the forecast for tomorrow?"); however, it is equally important to be aware of the set of behaviors that specifically contribute to that person's well-being so that the person can know what they might do differently to affect a different outcome. Understanding these factors will do more to guide prevention and early intervention efforts.

Although the preceding studies have focused only on predicting well-being, others have studied how certain daily behaviors affect well-being. For example, in an 8,000-person study, Stubbe et al. [28] showed that exercise participation is associated with higher levels of well-being. In addition, Wang et al. [30] demonstrated that students who slept less were more likely to be depressed, and a lower perceived stress score (PSS) was correlated with higher conversation frequency during the day. These studies investigate the stand-alone effects of each behavior on well-being. But these behaviors typically work together and do not affect well-being in isolation, with different combinations possibly resulting in different health outcomes. To that end, understanding how these factors work together could help provide insights into the groups of behaviors to target when looking to improve an individual's mental well-being.

Other researchers have used machine learning models to summarize human behavior. For example, using a correlation method, Phithakkitnukoon et al. [18] identified daily human activity patterns of eating, shopping, entertainment, and recreation from location estimates extracted from calls, messages, and Internet connections of 1 million users over a period of few months. In addition, LDA or topic models have been used to discover patterns underlying human behavior specifically as it relates to activity recognition. For example, Farrahi and Gatica-Perez [7] applied unsupervised topic models to large-scale location data from smartphones to discover daily human routine activities. Phung et al. [19] used LDA to infer the sequences of places a user routinely visits. LDA-based models have been used to discover daily routine patterns with data from wearable sensors [12, 27]. In addition, Ferrari and Mamei [9] used topic models to characterize mobility behaviors using location data collected from Google Latitude. These works demonstrate the potential of using probabilistic models to

\footnotetext{
${ }^{4}$ American College Health Assessment Spring 2013 and 2017 reports: http://www.acha-ncha.org/pubs_rpts.html. Accessed Jan. 10, 2019.
} 
summarize human behavior, but they only focus on mining activity patterns and do not have a direct application to improving health and well-being. Therefore, models that learn patterns of human behaviors with a specific goal of improving human health are important.

There is recent and growing interest in applying recommendation system approaches to health care problems, although work done in this area is still limited. Ge et al. [10] used a content-based method to recommend personalized healthy recipes for meals after users logged in and indicated meal and recipe preferences. Chomutare et al. [5] used a hybrid model to recommend peers to users who needed peer support to manage chronic diseases like diabetes; their approach was inspired by the "patients-like-me" concept [31]. These methods require the user to extensively rate each of the previously seen items, and then recommendations of new items are made based on user ratings.

Rabbi et al. [22] used a multi-armed bandit algorithm to make sequential decisions about which food a user should eat or which physical activity a user should perform to remain healthy. In this work, users were required to enter data manually about their eating habits, and the application mined users' physical activities from logged mobility patterns. The suggestions were contextual in nature and only depended on the user's history. Farrell et al. [8] used a heuristics-based approach to make recommendations on how to use physical activity and proper meal choices to reduce weight. The method used data from the user's daily meal and physical activity logs. A major drawback of these approaches is that users can easily become dissatisfied with a system that repeatedly makes familiar suggestions and recommends routine behaviors. Even though personalization is important, there could be value in learning and recommending healthy behaviors from similar individuals in a population, as well as customizing these suggestions based on data and preferences communicated from each person.

Thus, we would like to predict a person's well-being and then recommend evidence-based healthy behaviors that can help improve that person's state of well-being, if needed. However, this is difficult to implement, as it would ultimately require studies that prove casuality-and that include the collection of a plethora of data from that individual, and a manual rating for each behavior (not just a score at the end of day). Nevertheless, meaningful representations of a person's health behavior learned by the sLDA model affords us an opportunity to identify behaviors that have been associated with improved well-being for a person (or people like them) in the past, and which may be worth trying to adopt for improved health and well-being in the future. In the following, we describe our framework for carrying out this work.

\section{OUR FRAMEWORK}

We propose a framework for uncovering patterns of modifiable health behaviors that may influence the health and well-being of individuals. We define modifiable human behaviors as health behaviors that an individual can change, such as the time of going to bed. The current approaches to modeling human behavior either summarize routine behavior or model physical activity. Our work differs from these approaches because we focus on learning patterns of modifiable health behaviors that are predictive of well-being as opposed to only discovering structure in activity sensor data or finding topics that summarize various activities in different settings. We then illustrate how to use these patterns to provide actionable insights to individuals looking to improve their well-being.

Figure 1 summarizes our framework. First, in the collection stage, a broad range of multi-modal behavioral data is gathered from smartphones, sleep and activity monitors, and self-reported activities over a continuous and extended time (up to 30 days per person in this study). These data provide signals of modifiable health behaviors that are relevant to both mental and physical health. Second, the raw data collected is processed into feature representations of health behaviors. These representations and self-reported well-being measures serve as input features and labels, respectively, to an interpretable machine learning model. Third, the machine learning model is used to uncover latent patterns of health behaviors that may influence well-being. And last, these learned patterns are used to provide personalized evidence-based recommendations and actionable insights to individuals seeking to improve their well-being. Section 5.3 details how we make these recommendations, as well as the considerations we make. 

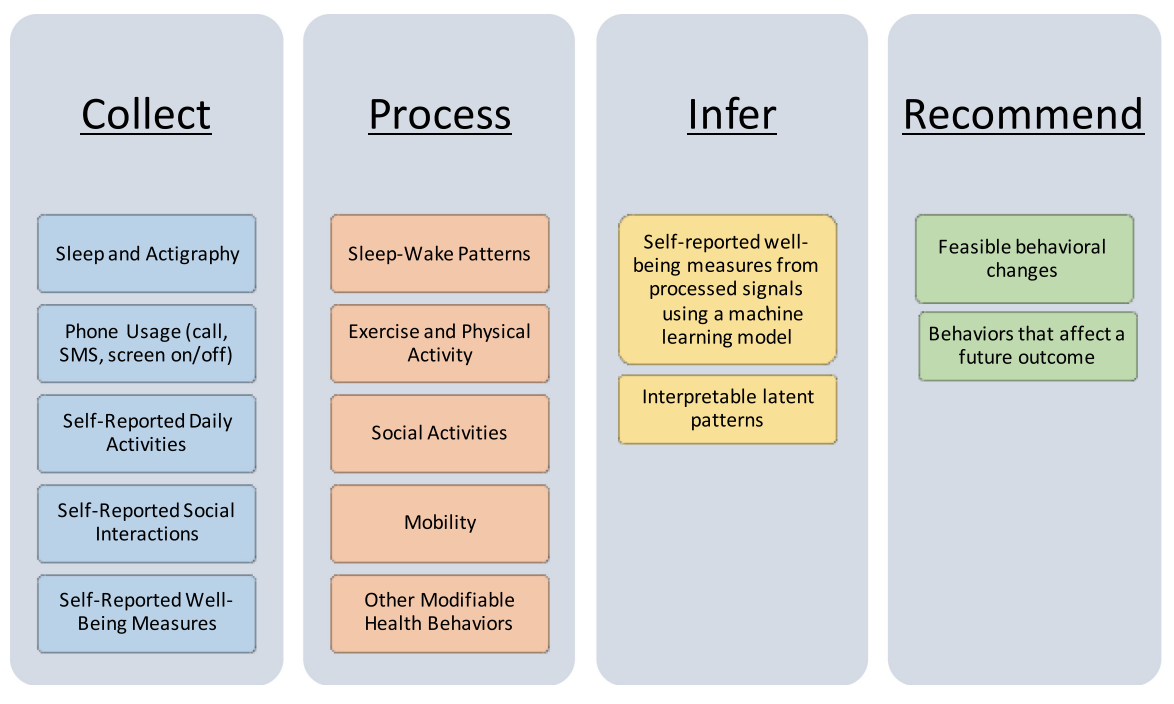

Fig. 1. Framework for assessing and recommending healthy behaviors for well-being improvement.

Our approach to modeling human behavior is novel and important for several reasons. First, our novel use of modifiable behaviors is significant because the insights discovered can provide individuals with an opportunity to change their behavior and potentially improve their well-being. In Section 5.1, we introduce a method of extracting these behaviors from multi-modal data and binning them to ensure that they are descriptive of a participant's day. Second, in learning patterns of health behaviors, we present a Bayesian model that creates meaningful and interpretable representations of these health behaviors. Representation learning can be characterized as finding representations of data that contain useful information toward some goal [1]. In this work, our goal is to create representations that are both useful for predicting future well-being and health states and helpful in recommendation tasks. Third, it is important to study how combinations of behaviors may influence well-being. As discussed previously, past research tends to learn associations between single health behaviors and well-being. But well-being is complex to model, and a behavior like a short night's sleep might be compensated for by good social interaction; it is thus important to examine how multiple behaviors work together to influence mood, stress, and health.

In our previous work, we used part of this novel framework to model self-reported stress [15]. In this article, we extend the framework to address other measures of well-being: self-reported mood (sad-happy) and self-reported health (sick-healthy). We show how the interpretable machine learning model can aid in providing actionable insights and recommendations to individuals looking to improve their well-being. This knowledge also provides an opportunity to get insights into influential variables that can become candidates for future causal studies. In this work, we will also show two case studies to illustrate how information from the sLDA model can be used to provide actionable insights to individuals looking to improve their stress, mood, and overall physical health.

\section{DATASET AND FEATURES}

We use data from the SNAPSHOT study that monitored students for 30 days over the course of six semesters, Fall 2013 to Spring 2016; in Spring 2017, students were monitored all semester long. For the 30-day study, about 50 students were enrolled each semester. In this work, we use a portion of the data from the 30-day study.

Various modalities contribute measurements to this dataset. They include pre- and post-study questionnaires, wearable sensors (Actiwatch and Q-sensor to collect activities and physiological data, respectively), mobile 
phones to log location, SMS, call and screen activities, and daily weather information. Participants filled out twice-daily surveys during the study, in the morning and evening. Information was collected about academic, extracurricular, and exercise activities, sleep, caffeine intake, and social interaction. Each morning and evening, the students were asked to rate on a visual analog scale of 0 to 100 their level of stress $(0=$ stressed-out; $100=$ calm-relaxed), their mood ( $0=$ sad; $100=$ happy), and their overall health $(0=$ sick; $100=$ healthy). The values from these rating scales reported in the evening constitute the self-report well-being labels used in this work for stress, mood, and health, respectively. In this work, we used data from only the following modalities: Actiwatch, Mobile Phone, and Behavioral surveys. Although modalities such as autonomic physiology and weather have contributed in earlier studies to improving prediction accuracy, we do not examine them in this work because they are not easily modifiable by participants. For more details about the dataset, see Sano [24] and Sano et al. [26].

\subsection{Data Representation and Features}

In this section, we discuss the selected modifiable behaviors and then present how to map the data to feature representations suitable for the modeling task. This constitutes the "process" part of our framework.

Selection of modifiable behaviors. We define modifiable behaviors as actions that can be controlled by the study participants, such as sleep duration or phone usage. Information was extracted from smartphone logs, wearable sensors, GPS coordinates, and behavioral surveys obtained from the participants during the 30-day study. We note that varying degrees of a particular behavior can lead to different outcomes. For example, sleeping 8 hours at night might lead to a participant reporting happy mood, whereas sleep deprivation might lead to a sad mood report from the same participant; these are two different behaviors, and we treat them as such. To be very specific when describing each day of data, we binned the behaviors based on duration, time of day, or number of occurrences. We did not bin behaviors that had binary yes/no response values. This led to a sparse representation of the data that is preferable when using topic models. We selected the number of bins for each feature by carefully examining its distribution and looking for natural breaking points. This process resulted in 134 behaviors. Table 1 shows the selected behaviors and their various bins [15].

"Bag-of-behaviors." Next, we converted the data into features for the model. Following the bag-of-words representation commonly used to model documents, we used a one-hot encoding to represent the binned modifiable behaviors in each participant's day: $x \in\{0,1\}^{|V|}$, where $V$ is the set of modifiable behaviors used in the model, 0 represents absence, and 1 represents the presence of the modifiable behavior in that participant's day. Since we want to learn how combinations of behaviors affect self-reported well-being, each individual's day is represented by the group of specific behaviors present. This is an un-ordered representation of the behaviors, but binning allows us to retain some of that information. We show that this feature representation allows for interpretable latent patterns.

We threw out days with fewer than 15 behaviors present because they were insufficient to train the model. In total, we ended up with 5,397 days of data and 115,659 observations from 224 unique participants across six semesters.

Response variables. Every morning and evening, the participants self-reported their perceived stress (stressedcalm), mood (sad-happy), and health (sick-healthy) on a visual analog scale of 0 to 100 , with 0 indicating the worst level of stress, sadness, or sickness, and 100 the highest level of calmness, happiness, or health. Figure 2 shows the frequency of the raw values of these response variables. We see that these labels are not uniformly distributed in the data but are skewed toward a healthier population. This is in line with our goals of understanding behavioral influences on well-being in a relatively healthy population.

In psychology, self-reports are currently the gold standard for assessing well-being; however, in this work, we learn associations between these self-reports and combinations of behaviors that can be measured objectively. Thus, we provide evidence-based insights about behaviors that an individual might consider to modify if they wish to improve their well-being.

ACM Transactions on Computing for Healthcare, Vol. 1, No. 1, Article 4. Publication date: February 2020. 
Table 1. Modifiable Behaviors, Bins, and Total Number of Days the Behaviors Were Reported by the Participants

\begin{tabular}{|c|c|c|c|}
\hline Modality & Behaviors & Bins & Total Num. of Days \\
\hline \multirow{5}{*}{ Call } & Num. total outgoing calls & $0,1,2,3-4, \geq 5$ & 3,911 \\
\hline & Num. unique outgoing calls & $0,1,2, \geq 3$ & 3,911 \\
\hline & Num. unique missed calls & $3-4, \geq 5$ & 528 \\
\hline & Duration incoming calls (all day) (min.) & $0,0-2,2-4,4-6,6-12, \geq 12$ & 3,911 \\
\hline & Duration outgoing calls (all day) (min.) & $0,0-2,2-4,4-6,6-12, \geq 12$ & 3,911 \\
\hline \multirow{4}{*}{ Screen } & Num. on/off events (5PM-midnight) & $0,0-25,25-50,50-75$ & 5,040 \\
\hline & Duration on/off events (all day) (hours) & $0,0-2,2-3,3-4, \geq 4$ & 5,064 \\
\hline & Duration on/off events (midnight-3AM) (hours) & $0,0-0.5, \geq 0.5$ & 5,057 \\
\hline & Duration on/off events (5PM-midnight) (hours) & $0,0-0.5,0.5-1,1-2, \geq 2$ & 5,040 \\
\hline \multirow{2}{*}{ SMS } & Num. unique outgoing SMS (all day) & $0,1,2,3-4,5-10, \geq 10$ & 5,071 \\
\hline & Num. unique outgoing SMS (5PM-midnight) & $0,1,2-5, \geq 5$ & 5,071 \\
\hline \multirow{8}{*}{ Actiwatch sleep } & Duration today's sleep (hours) & $1-4,4-6,6-7,7-8,8-10, \geq 10$ & 4,371 \\
\hline & Duration yesterday's sleep (hours) & $1-4,4-6,6-7,7-8,8-10, \geq 10$ & 3,999 \\
\hline & Duration day before yesterday's sleep (hours) & $1-4,4-6,6-7,7-8,8-10, \geq 10$ & 3,841 \\
\hline & Bedtime (hour of the day) & 12-1AM, 1-2AM, 2-3AM, & 4,277 \\
\hline & & 3-4AM, 4-6AM, 6-10AM, & \\
\hline & & $10 \mathrm{AM}-8 \mathrm{PM}, 8-11 \mathrm{PM}$ & \\
\hline & Bedtime deviation from participant's mean (hours) & $\leq-2,-1,0,1, \geq 2$ & 4,371 \\
\hline & Weekly sleep regularity & $\begin{array}{l}0-0.4,0.4-0.5,0.5-0.6,0.6-0.7 \\
0.7-0.8,0.8-0.9,0.9-1\end{array}$ & 4,363 \\
\hline \multirow{3}{*}{ Location } & Time on campus (hours) & $0,0-1,1-8, \geq 8$ & 3,716 \\
\hline & Time indoors (hours) & $0,0-2,3-5,6-7,8,9-10, \geq 10$ & 3,716 \\
\hline & Time outdoors (hours) & $0,0-1,1-8, \geq 8$ & 3,716 \\
\hline \multirow{8}{*}{ Survey activities } & Study duration (hours) & $0,0-2,2-4,4-6,6-8, \geq 8$ & 5,397 \\
\hline & Exercise duration (hours) & $0,0-1,1-2, \geq 2$ & 5,397 \\
\hline & Extracurricular duration (hours) & $0,0-2,2-4, \geq 4$ & 5,397 \\
\hline & Academic duration (hours) & $0,0-2,2-3,3-4,4-6, \geq 6$ & 5,397 \\
\hline & Naps $(0 / 1)$ & & 1,084 \\
\hline & All-nighter (0/1) & & 141 \\
\hline & Caffeine consumption $(0 / 1)$ & & 2,029 \\
\hline & Alcohol consumption $(0 / 1)$ & & 666 \\
\hline \multirow{4}{*}{ Survey interaction } & Negative social interaction $(0 / 1)$ & & 555 \\
\hline & Positive social interaction $(0 / 1)$ & & 1,439 \\
\hline & Pre-sleep in-person interaction $(0 / 1)$ & & 1,961 \\
\hline & Pre-sleep media interaction $(0 / 1)$ & & 1,961 \\
\hline
\end{tabular}

As a pre-processing step, we log-transformed the labels to achieve approximate normality as required by the model (see Section 5.1). These real-valued log-transformed stress labels, $y \in \mathbb{R}$, are the response variables in the supervised model. Our modeling goal is to find latent patterns that are best predictive of these real-valued labels.

In our experiments, we also trained the model using other kinds of response variable transformations: $z$-transformed the labels (normalized), divided each response by 100 (scaled), centered the scaled labels at 0 by 


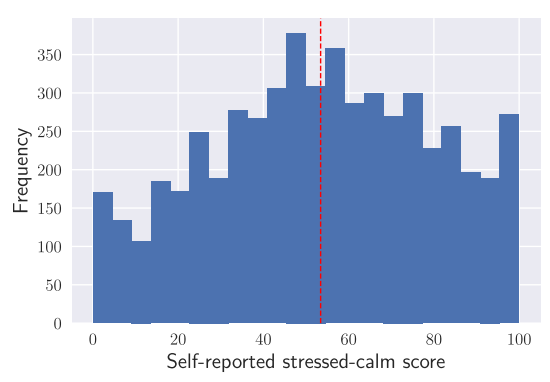

(a) Stressed-calm

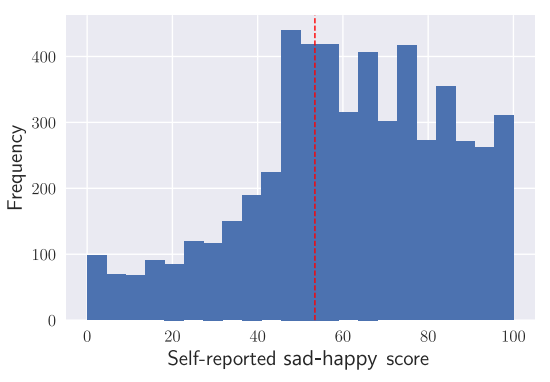

(b) Sad-happy

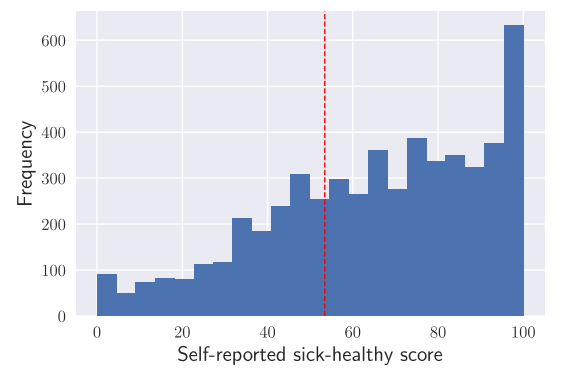

(c) Sick-healthy

Fig. 2. Frequency of self-reported stressed-calm $($ mean $=53.43)(a)$, sad-happy $($ mean $=60.96)(b)$, and sick-healthy $($ mean $=$ $65.00)$ (c) response variables. Their respective means are shown in the vertical red line.

subtracting the mean (scaled-shifted), log-transformed the scaled labels (scaled-log), and centered the logtransform at 0 by subtracting the mean (log-shifted). However, none of these other response transformations performed as well as the log-transform in the prediction task. We omit the results for brevity.

\section{METHODS}

In this section, we discuss the methods used in the "infer" and "recommend" stages in our framework. First, we describe the supervised topic model or latent Dirichlet allocation (sLDA) used to uncover the latent patterns of modifiable human behaviors. Then, we illustrate how the inferred latent patterns can be used to provide evidence-based insights to individuals.

\subsection{Supervised Topic Models}

Topic models are used to learn the probability distribution of words in collections of documents in an unsupervised manner [4]. However, the goal of this work is to use topic models to learn patterns that maximize the likelihood of combinations of behaviors present in the participants' days leading to healthy outcomes. Although unsupervised models are sufficient to summarize words in a document, they are not ideal for real-world problems such as modeling health behaviors that may influence well-being. If we use an unsupervised model, we will likely discover broad patterns that run throughout the days. Health behaviors are dynamic and vary over the time of day and across people. Hence, since we want patterns that are predictive of an outcome such as well-being, supervision is required. In this work, we use the sLDA model, proposed by Blei and McAuliffe [3], to assess health behaviors. Each day has three response variables external to the behaviors present, and the aim is to find patterns that are best predictive of each of the response variables. To do this, we model each of the response variables separately. 


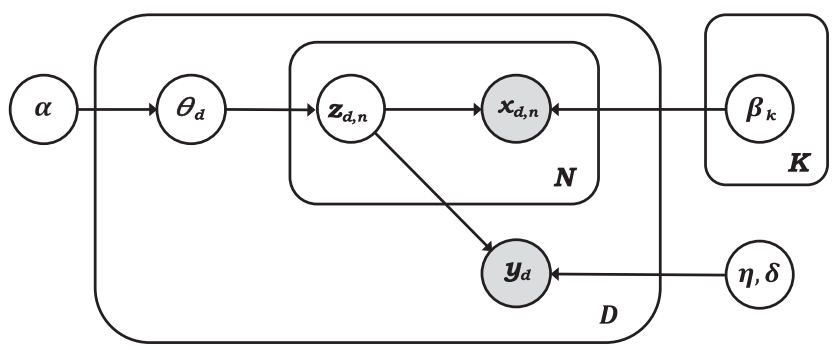

Fig. 3. Graphical model representation for the sLDA model. Shaded nodes are observed, and arrows indicate dependencies between variables [3].

We use $d \in 1, \cdots D$ to index the number of days present in the dataset, $n \in 1 \cdots N$ to index the number of behaviors observed on day $d$, and $k \in 1, \cdots K$ to index the number of latent patterns. In the model, each day is represented as a collection of discrete random variables $x_{1: N}$; these are the observed 134 behaviors. The unobserved patterns $\beta_{1: K}$ are probability distributions over the set of 134 health behaviors observed in the data. We assume that the same set of $K$ latent patterns, $\beta_{1: K}$, generate the behaviors we observe in all of the days present in the dataset, but these patterns have different proportions $\theta$ on different days. The different proportions of these patterns give rise to different combinations of behaviors each day and thus to different responses.

Following sLDA, we jointly model each day's observed behaviors $x_{1: N}$, and response variable $y$ to find latent patterns that are best predictive of the stress, mood, or health responses of unseen days. Given the Dirichlet parameter $\alpha$, response parameters $\eta$ and $\delta$, and fixed latent patterns $\beta_{1: K}$, the behaviors on each day $d$ and corresponding response variable $y$ come from the following data-generative process:

$$
\begin{aligned}
\theta \mid \alpha & \sim \operatorname{Dir} .(\alpha) & & \text { (pattern proportions) } \\
z_{n} \mid \theta & \sim \operatorname{Mult} .(\theta) & & \text { (pattern assignment for each behavior) } \\
x_{n} \mid z_{n}, \beta_{1: K} & \sim \operatorname{Mult}\left(\beta_{z_{n}}\right) & & \text { (observed behavior) } \\
y \mid z_{1: N}, \eta, \delta & \sim \mathcal{N}\left(\eta^{T} \bar{z}, \delta\right) & & \text { (response variable), }
\end{aligned}
$$

where

$$
\bar{z}:=\frac{1}{N} \sum_{n=1}^{N} z_{n}
$$

represents the unobserved empirical pattern frequencies that occurred on day $d$ and $z_{n}$ is an indicator variable. Figure 3 presents a graphical model representation of the sLDA.

Following Blei and McAuliffe [3], each of the response variables $y$ is modeled as a Gaussian linear model, with regression coefficients $\eta$ and covariates $\bar{z}$. By regressing $y$ on the frequencies $\bar{z}$, we bind the behaviors expressed on each day (and the night before) $d$ to the response variables reported on that day. This is a reasonable approach because it limits the latent patterns to explain both the behaviors present and the corresponding response variable reported on that day. This effectively ensures that the learned patterns are best predictive of the response $y$. The mean and variance parameters of the Gaussian are $\eta^{T} \bar{z}$ and $\delta$, respectively.

5.1.1 Variational Expectation-Maximization. The latent variables are the pattern proportions $\theta$ and each behavior's pattern assignment $z_{n}$. In this model, $\beta_{1: K}, \eta$, and $\delta$ are constants to be estimated, and $\alpha$ is a fixed hyper-parameter. To learn the latent variables from the training data $\left\{x_{d, 1: N}, y_{d}\right\}_{d=1}^{D}$, we approximate the posterior $p\left(\theta, z_{1: N} \mid x_{1: N}, y, \alpha, \beta_{1: K}, \eta, \delta\right)$ via variational inference. Following Blei and McAuliffe [3], the fully factorized 


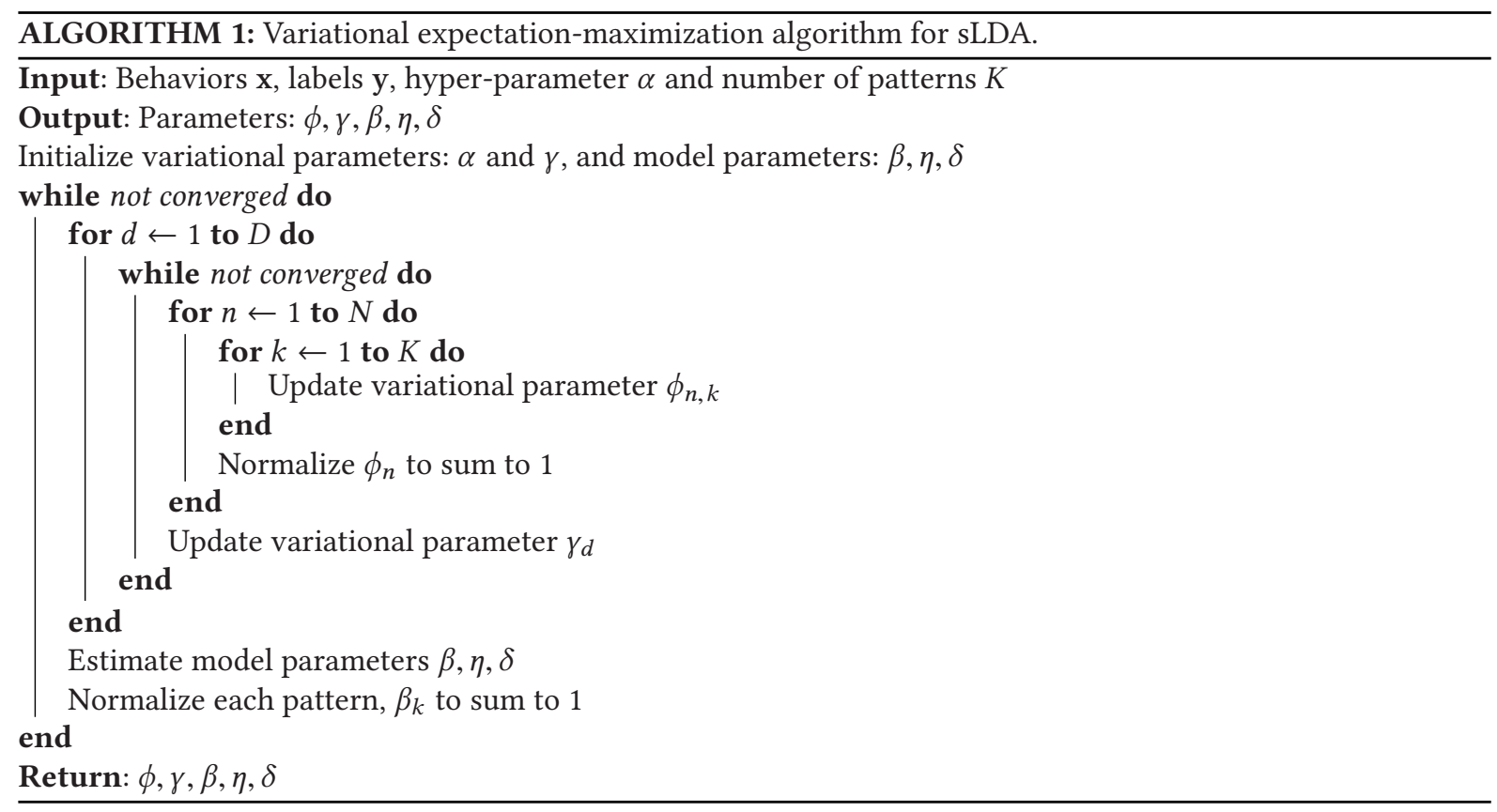

variational distribution that approximates the posterior for each day is specified as

$$
q\left(\theta, z_{1: N} \mid \gamma, \phi_{1: N}\right)=q(\theta \mid \gamma) \prod_{n=1}^{N} q\left(z_{n} \mid \phi_{n}\right)
$$

where $\gamma$, a $K$-dimensional Dirichlet parameter vector, learns the latent pattern proportions for each day $d$, and $\phi_{n}$, a $K$-dimensional categorical parameter vector, learns the assignment probabilities of behavior $n$ to each of the $K$ patterns. The vector $Z_{n}$ (representing the topic assignment $z_{n}$ in practice) is a $K$-dimensional indicator vector, with the non- zero entry corresponding to the pattern assigned to behavior $n$; thus,

$$
\mathrm{E}\left[Z_{n}\right]=q\left(z_{n}\right)=\phi_{n}
$$

and

$$
\mathrm{E}[\bar{Z}]=\bar{\phi}=\frac{1}{N} \sum_{n=1}^{N} \phi_{n}
$$

After learning the posterior, we estimate the model parameters $\left(\beta_{1: K}, \eta, \delta\right)$ using variational expectationmaximization. The parameters are chosen such that the following evidence lower bound (ELBO) is optimized:

$$
\mathcal{L}\left(\alpha, \beta_{1: K}, \eta, \delta ;\left\{x_{d, 1: N}, y_{d}\right\}_{d=1}^{D}\right)=\sum_{d=1}^{D} \mathrm{E}_{d}\left[\log p\left(\theta_{d}, z_{d, 1: N}, x_{d, 1: N}, y_{d}\right)\right]+\mathrm{H}\left(q_{d}\right),
$$

where $\mathrm{H}\left(q_{d}\right)$ is the entropy of each day's variational distribution $q_{d}$.

In the E-step, we estimate the posterior distribution for each day-response pair by updating $\gamma$ and each of the $\phi_{n}$. And in the M-step, we update the model parameters $\beta_{1: K}, \eta, \delta$ to optimize the ELBO. The update equations are outlined in Blei and McAuliffe [3]. The algorithm is summarized in Algorithm 1. 
5.1.2 Prediction. Once we have a fitted model, the pattern proportions $\gamma^{\text {new }}$, and behavior assignment probabilities $\phi^{\text {new }}=\left\{\phi_{n}\right\}_{n=1}^{N_{d, \text { new }}}$, of any previously unseen day are re-estimated conditioned on the current estimate of the model parameters $\beta, \eta, \delta$ until convergence. This step is done iteratively until convergence. Then, we predict the day's response as follows:

$$
\mathrm{E}\left[y \mid x_{1: N}, \alpha, \beta_{1: K}, \eta, \delta\right] \approx \eta^{T} \mathrm{E}_{q}[\bar{z}]=\eta^{T} \bar{\phi}_{\text {new }}
$$

where the expectation is taken with respect to the variational distribution $q$. With this, the model can predict any new participant's self-reported well-being and also learn the day's latent pattern proportions.

\subsection{Experiments}

In the previous section, we explained that we use sLDA to jointly model the behaviors and each of the response variables separately. This results in three different set of latent patterns that are best predictive of the stress, mood, and health response, respectively. To test its performance, we compared sLDA to two baseline approaches:

- A linear regression analysis on the $\bar{\phi}_{d}$ learned from unsupervised LDA. This is the same as using LDA patterns as prediction features. We call this the $L D A+$ regression $(\mathrm{LDA}+\mathrm{R})$ approach. Since we hypothesize that the sLDA will find better predictive patterns, we expect it to perform better than LDA+R approach.

- An $\mathrm{L}_{1}$-regularized least-squares regression analysis, also known as Least Absolute Shrinkage and Selection Operator (LASSO), using each day's estimated distribution over behaviors as the model covariates (i.e., normalizing the features to sum to 1). The LASSO has a feature selection property, and hence it is widely used in high-dimensional problems. In addition to comparing its performance results with the SLDA, we are also interested in examining the features that were selected by the LASSO model for the three (stress, mood, and health) well-being regression tasks.

For the sLDA analysis, we initialized $\beta_{1: K}$ to randomly perturbed uniform patterns; $\delta$ to the variance of the $\log$-transformed stress, mood, or health responses, respectively; and $\eta$ to a $K$-dimensional vector of zeros (we found that initializing $\eta$ to a vector of ones provided comparable results). In addition, we fixed $\alpha=1 / K$. In the E-step, we ran coordinate ascent variational inference (CAVI) until the relative change in each day's ELBO was less than 0.001, indicating convergence. We ran the M-step until the relative change in the overall likelihood bound was less than 0.0001 . For the LDA model, we initialized $\beta_{1: K}$ similarly and used the same convergence criteria and value for $\alpha$.

We applied fivefold cross validation to the dataset and quantified the model's performance in two ways. First, for each of the well-being measures, we computed a binary prediction accuracy and F1 scores on the held-outfold predictions, which measured how well the learned patterns predicted high versus low values. To convert to binary labels, true and predicted response variables equal to or greater than the respective means were considered high values (label 1), and values below the mean were considered low values (label 0 ). We also computed binary prediction accuracy results using a value of 50 for self-reported stress labels as the threshold. Since the mood and health labels are more skewed toward the right, we do not show results for this threshold. In addition, we report the same performance metrics on the held-out-fold predictions for the LDA+R approach. We assessed the prediction quality of these models over different numbers of latent patterns, 2-20.

For the LASSO model, we used fivefold cross validation to select the optimal regularization parameter and report the highest mean ( \pm standard deviation) correlation and binary prediction accuracies achieved on the held-out fold. We compare these values to the highest value sLDA achieved across the different numbers of patterns. 


\subsection{Recommending Health Behaviors}

In making health behavior recommendations, it is important to take the following considerations into account. First, recommended behaviors should be feasible-that is, these behaviors should only require slight modifications to an existing routine, so as not to burden a person who may be feeling stressed, sad, or unhealthy. This would mean recommending behaviors from similar days with better outcomes, making it likelier that fewer changes can lead to better results. For each day, sLDA learns personalized probability distributions $\gamma^{\text {new }}$ over the latent patterns $\beta_{1: K}$ and thus is able to capture similarities in the behaviors present in participant-days. Second, since the scores are reported in the evening, it is important to recommend behaviors that are both achievable and that might improve a future outcome, such as changing the bedtime, length of sleep, and social interactions planned for the following day. Finally, when finding similar days, it is beneficial to include data from other participants. This is because currently available data is insufficient to train an sLDA model for each person. In addition, if a person only has unhealthy behaviors, it would be impossible to recommend a healthy behavior based only on the person's existing data. Including behaviors from others also ensures that participants do not get bored from being recommended the same recycled behaviors from their past.

As discussed in Section 5.1.2, given behavior data from a previously unseen day, $x_{1: N}$, the fitted sLDA model is able to learn the probability distribution of the latent patterns present $\gamma^{\text {new }}$, and the pattern assignments for each behavior $n, \phi^{\text {new }}=\left\{\phi_{n}\right\}_{n=1}^{N_{d, \text { new }}}$, by iteratively re-estimating the update equations. Once these latent variables are learned, the model can now compute the expected well-being score for the day, $\mathrm{E}\left[y \mid x_{1: N}, \alpha, \beta_{1: K}, \eta, \delta\right]$. Given $\gamma^{\text {new }}$, we can find other participant-days closest to it but with higher self-reported well-being scores. We do this by comparing $\gamma^{\text {new }}$ estimated by the sLDA model to the pattern distributions of other days used to train the model. There are several similarity metrics that can be used for this comparison, but we select the Jensen-Shannon (JS) distance.

5.3.1 JS Distance. The JS distance is derived from the Jensen-Shannon divergence (JSD). JSD is a method of measuring the similarity between two probability distributions, and it is based on the KL-divergence. However, unlike the KL-divergence, JSD is symmetric and always finite. Its symmetry ensures that the similarity between distributions $P$ and $Q$ is the same as the similarity between distributions $Q$ and $P$ (i.e., $J S D(P \| Q)=J S D(Q \| P)$ ). In addition, when the natural $\log$ is used, JSD is bounded as follows: $0 \leq J S D \leq 1$.

For two discrete distributions $P$ and $Q$, JSD is defined as

$$
J S D(P \| Q)=\frac{1}{2} D(P \| M)+\frac{1}{2} D(Q \| M),
$$

where

$$
M=\frac{1}{2}(P+Q),
$$

and $D$ is the KL-divergence

$$
D(P \| Q)=\sum_{i} P_{i} \log \frac{P_{i}}{Q_{i}}
$$

We have that

$$
J S D(P \| Q)=\frac{1}{2} \sum_{i}\left[P_{i} \log \left(\frac{P_{i}}{\frac{1}{2}\left(P_{i}+Q_{i}\right)}\right)+Q_{i} \log \left(\frac{Q_{i}}{\frac{1}{2}\left(P_{i}+Q_{i}\right)}\right)\right],
$$

and the JS distance is $\sqrt{J S D(P \| Q)}$.

The smaller the JS distance, the more similar two distributions are, and in our case, the more similar participant-days are. When minimizing the JS distance, we constrain the model to find similar participant-days with higher self-reported scores. In Section 6.3, using two case studies, we illustrate how we can make recommendations to two different participants in the SNAPSHOT study using sLDA and the JS distance. 


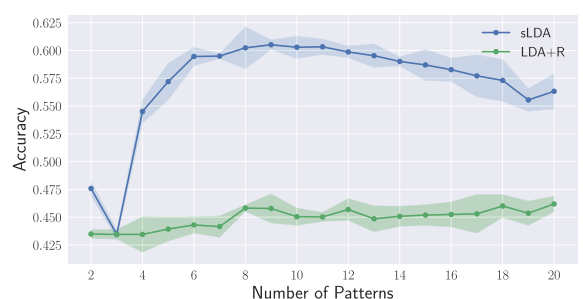

(a) Stressed-calm

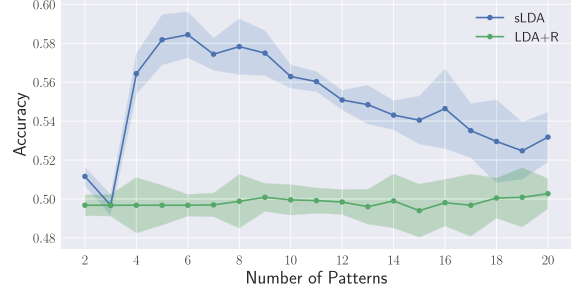

(b) Stressed-calm

Fig. 4. Average binary prediction accuracy (over five runs, \pm standard deviation) for sLDA and LDA+R models across different patterns for self-reported stress when threshold $=50(\mathrm{a})$ and threshold $=53.43(\mathrm{~b})$.

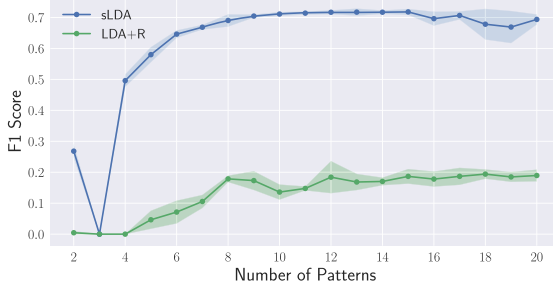

(a) Stressed-calm

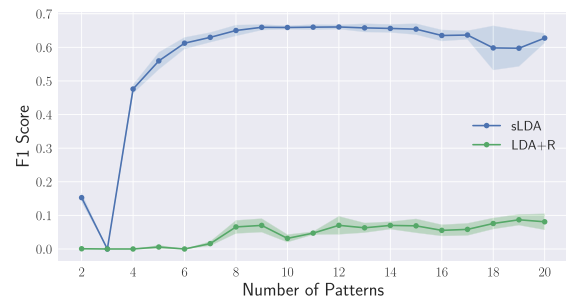

(b) Stressed-calm

Fig. 5. Average F1 score (over five runs, \pm standard deviation) for sLDA and LDA+R models across different patterns for self-reported stress when threshold $=50$ (a) and threshold $=53.43(\mathrm{~b})$.

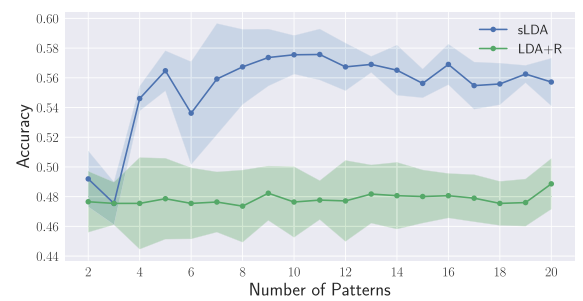

(a) Sad-happy

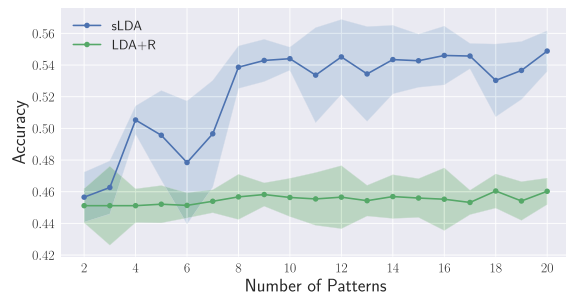

(b) Sick-healthy

Fig. 6. Average binary prediction accuracy (over five runs, \pm standard deviation) for sLDA and LDA+R models across different patterns for self-reported mood when threshold $=60.96$ (a) and self-reported health when threshold $=65.00$ (b).

\section{RESULTS AND DISCUSSION}

The average binary prediction accuracies and F1 scores (over five runs) for both thresholds, 50 (self-reported stress only) and the mean stress response (53.43), are shown in Figures 4, 5, 6, and 7, respectively. Remember, when reviewing these results, that our goal is not to build the best model for a general well-being recognition problem, and thus we do not compare different machine learning models. (Nor do we use other features that may improve prediction accuracy.) Instead, the challenge is to identify sets of modifiable behaviors (patterns) that are significant in helping predict stress, mood, or health, and evaluate if they are better than a control set (the unsupervised patterns found by LDA).

Our results indicate that across multiple numbers of patterns and the three different well-being measures, the latent patterns from the sLDA model outperformed those from the LDA $+\mathrm{R}$ model on all performance metrics. Note that patterns from the baseline unsupervised LDA model (LDA+R) perform even worse than a random 


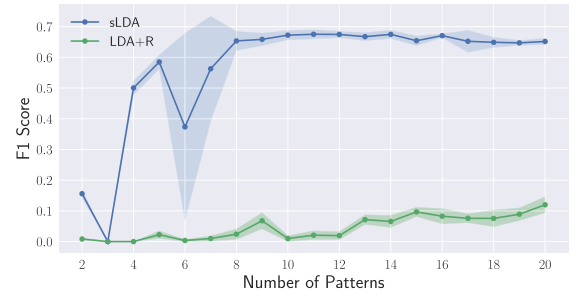

(a) Sad-happy

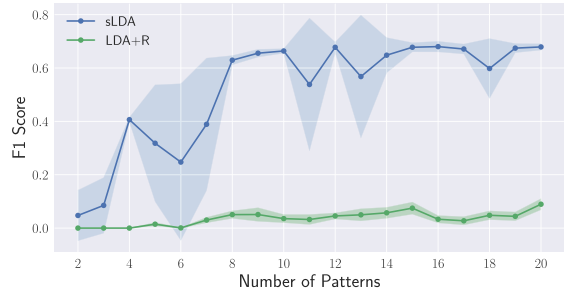

(b) Sick-healthy

Fig. 7. Average F1 score (over five runs, \pm standard deviation) for sLDA and LDA+R models across different patterns for self-reported mood when threshold $=60.96$ (a) and self-reported health when threshold $=65.00$ (b).

Table 2. Mean ( \pm Standard Deviation) of Binary Accuracies and F1 Scores for LDA+R, sLDA, and LASSO Across the Three Response Labels: Stress, Mood, and Health

\begin{tabular}{|c|c|c|c|c|c|}
\hline & & \multicolumn{4}{|c|}{ Performance Metrics } \\
\hline & Model & $\begin{array}{l}\text { Binary Accuracy } \\
\text { (thresh. = mean) }\end{array}$ & $\begin{array}{c}\text { Binary Accuracy } \\
(\text { thresh. }=50)\end{array}$ & $\begin{array}{c}\text { F1 Score } \\
\text { (thresh. = mean) }\end{array}$ & $\begin{array}{c}\text { F1 Score } \\
(\text { thresh. }=50)\end{array}$ \\
\hline \multirow{3}{*}{ Stressed-Calm } & sLDA & $58.4 \%( \pm 1.2)$ & $60.5 \%( \pm 0.4)$ & $0.66( \pm 0.01)$ & $0.72( \pm 0.01)$ \\
\hline & LASSO & $57.0 \%( \pm 1.2)$ & $56.5 \%( \pm 1.0)$ & $0.41( \pm 0.03)$ & $0.48( \pm 0.02)$ \\
\hline & $\mathrm{LDA}+\mathrm{R}$ & $50.3 \%( \pm 0.8)$ & $46.2 \%( \pm 0.7)$ & $0.09( \pm 0.02)$ & $0.19( \pm 0.01)$ \\
\hline \multirow{3}{*}{ Sad-Happy } & sLDA & $57.6 \%( \pm 1.7)$ & - & $0.68( \pm 0.02)$ & - \\
\hline & LASSO & $56.3 \%( \pm 1.7)$ & - & $0.42( \pm 0.01)$ & - \\
\hline & $\mathrm{LDA}+\mathrm{R}$ & $49.0 \%( \pm 2.0)$ & - & $0.12( \pm 0.03)$ & - \\
\hline \multirow{3}{*}{ Sick-Healthy } & sLDA & $54.6 \%( \pm 1.8)$ & - & $0.68( \pm 0.02)$ & - \\
\hline & LASSO & $49.4 \%( \pm 1.0)$ & - & $0.24( \pm 0.02)$ & - \\
\hline & $\mathrm{LDA}+\mathrm{R}$ & $46.0 \%( \pm 1.0)$ & - & $0.09( \pm 0.02)$ & - \\
\hline
\end{tabular}

Bold entries represent a statistically significant improvement of the SLDA model over LASSO $(p<.05)$.

predictor. This implies that sLDA learns latent patterns that are better at predicting the self-reported well-being measures compared to the patterns learned in an unsupervised way.

Table 2 shows the best mean ( \pm standard deviation) of the performance metrics the LASSO achieved, and the highest value LDA+R and sLDA achieved across the different number of patterns, across different response variables, and averaged over five repetitions. For the binary prediction accuracy metric, sLDA had modest improvements over LASSO of 7\%, 2.5\%, 2.3\%, and 10.5\%, when the thresholds were set at 50 and for the mean stress response, mean mood response, and mean health response, respectively. For the F1 score metric, sLDA had improvements over LASSO of in the range of 50\% to $183 \%$ across the thresholds and response variables. The LASSO model consistently outperformed the LDA+R approach. A Welch's $t$-test revealed that sLDA had a statistically significant improvement over LASSO's prediction accuracy when the binary data split threshold was set at 50 and for the mean health response. The sLDA also had statistically significant improvements over LASSO's F1 scores for both binary split thresholds and across all three response variables. We also note the advantage that sLDA models the latent structure of the data, making its representation useful for other purposes beyond prediction.

\subsection{LASSO}

Recall that LASSO has feature selection properties, selecting a subset of relevant features for the modeling tasks. We are interested in examining the behaviors that were selected as best predictors of self-reported stress, mood, and health in this cohort. Figures 8, 9, and 10 show the non-zero model coefficients for LASSO. In the study, the 


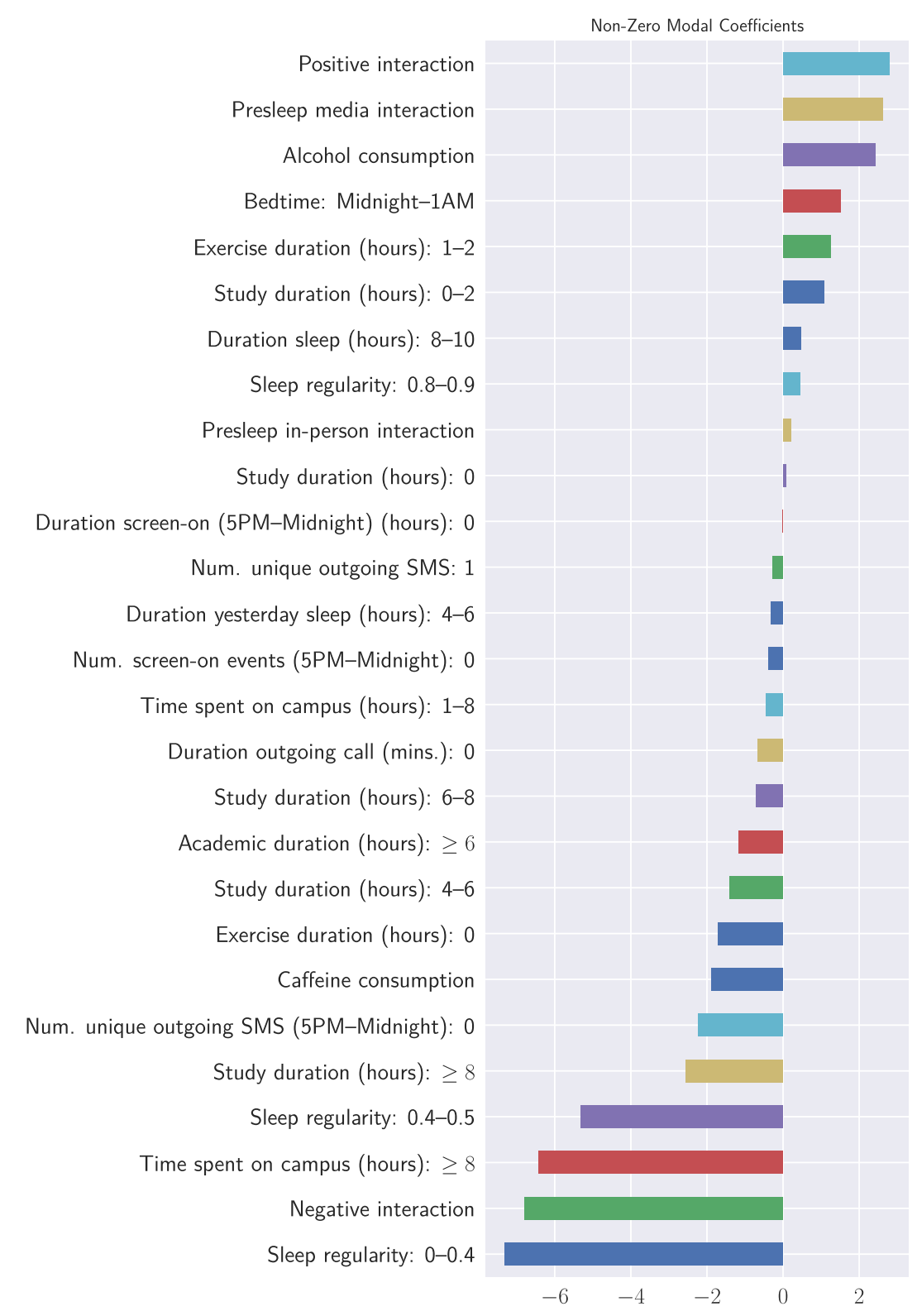

Fig. 8. LASSO non-zero coefficient estimates for self-reported stress. In the data, 100 represents the highest level of calmness and 0 represents the worst stress reported later in the evening. Thus, the positive coefficients are positively associated with increasing calmness.

self-reported labels were on a scale of 0 to 100 . The self-reported well-being levels were forecast for each night's labels using data from before that night. This means that the model selected features that were best predictive of increasing or decreasing well-being. We discuss some of the behaviors next.

Social interactions. Different types of social interactions had different effects on well-being. According to the models, the feature of self-reported in-person interactions just before sleep (the night before) was positively 


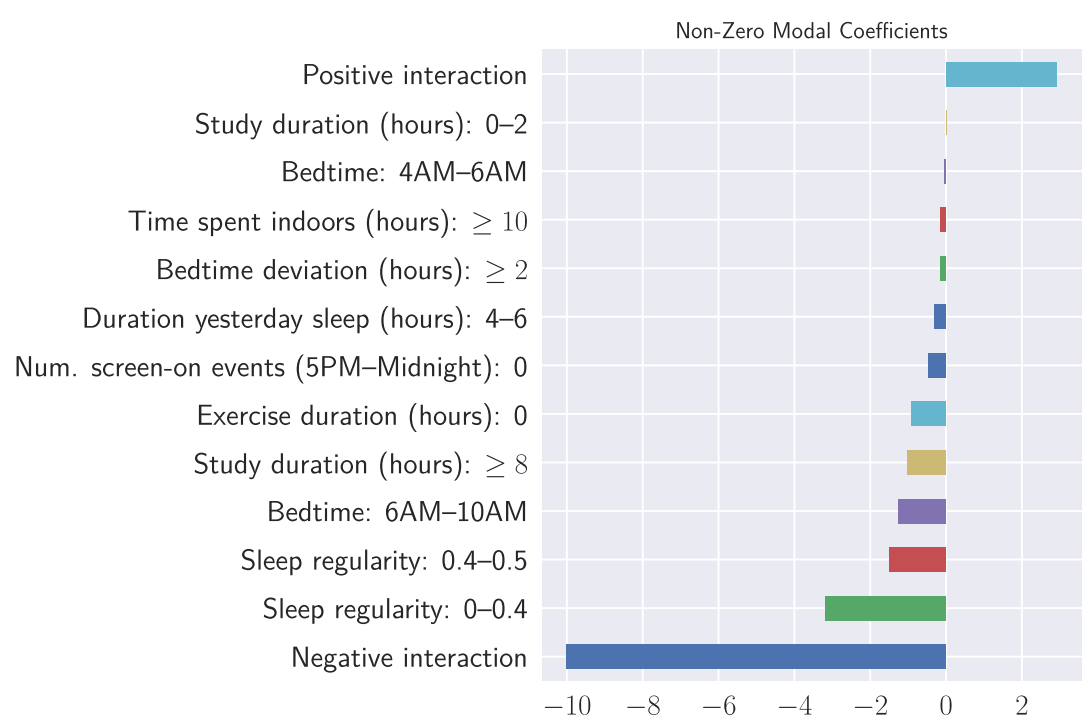

Fig. 9. LASSO non-zero coefficient estimates for self-reported happiness. In the data, 100 represents the highest level of happy mood and 0 represents the highest level of sad mood reported later in the evening. Thus, the positive coefficients are positively associated with increasing good mood.

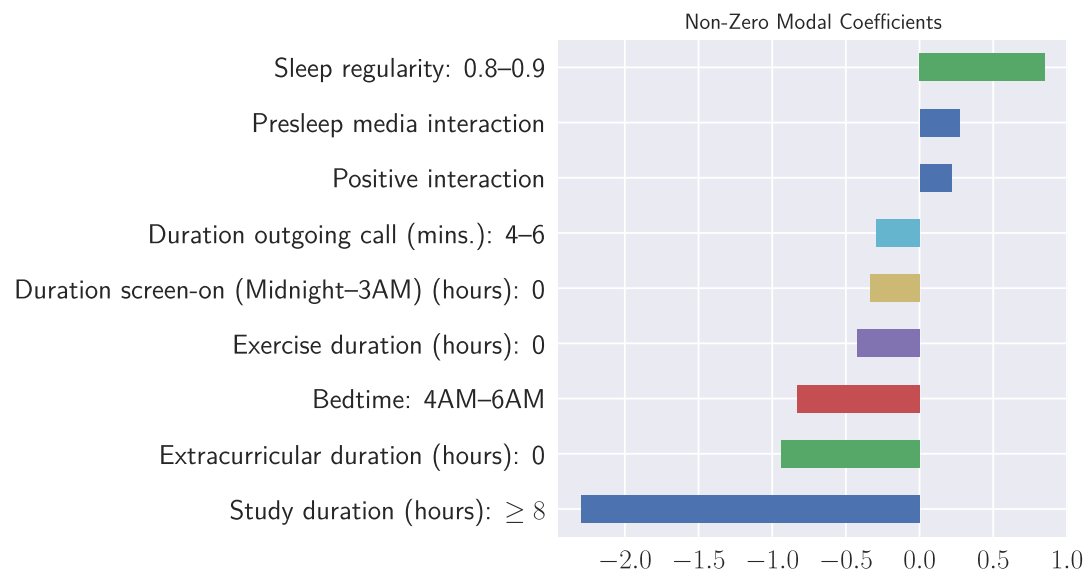

Fig. 10. LASSO non-zero coefficient estimates for self-reported health. In the data, 100 represents the highest level of health and 0 represents the highest level of sickness reported later in the evening. Thus, the positive coefficients are positively associated with increasing health.

associated with subsequent calmness for the upcoming evening. In addition, positive interactions during the day were positively associated with calmness and happy mood reported later that evening. There was also a positive association between positive interactions during the day and self-reported health later in the evening, albeit a smaller effect than the other two well-being measures. In contrast, negative interactions during the day were best predictive of stress and sad mood also reported later that evening.

Having few interactions through electronic devices, such as not initiating any calls during the day or not sending any text messages after 5PM, were high predictors of stress that evening. Moreover, having no screen-on events in the evening after 5PM was predictive of sad mood that same evening. This is consistent with research 
showing that students with low mental health, as measured by mental health composite scores (MCS), had a lower probability of interacting with electronic media prior to bedtime [26]. In addition, the StudentLife study by Wang et al. [30] found that students who had frequent conversations in the evening were less likely to be stressed ( $r=-0.386, p=.015)$ [30]. In addition to in-person interactions, we see that interaction with media (which could be entertainment) is a predictor of calmness or good health.

Bedtime, sleep duration, and sleep regularity. Sleep features had high importance in predicting all three wellbeing measures. Note that we attribute sleep the night before as today's behavior or feature. First, going to bed between midnight and 1AM (slightly earlier than the study participants' average of 2AM), and having about 8 to 10 hours of sleep the previous night, was predictive of a calm day (as reported at the end of day today). This finding aligns with results by Wang et al. [30] that showed a strong negative association between sleep duration and perceived stress $(r=-0.355, p=.024)$; students getting more sleep experienced less stress. However, going to bed much later between the hours of $4 \mathrm{AM}$ and 6AM had a negative effect on health and a slight negative effect on mood reported later that evening. Second, sleep regularity (SR), which captures changes in sleep timing on a day-to-day timescale, is a predictor of stress and calm even when controlling for sleep duration [25]. In this model, high SR (0.8-0.9) was highly predictive of calmness and health, and low SR (0-0.5) was highly predictive of stress and sad mood. This is consistent with a previous study that found that SR was associated with better well-being in college students [25]. We were also interested in seeing the effects of the previous two nights' sleep duration on today's outcome, so we included the sleep duration of those nights as behaviors in our model. For example, if we have a self-reported calm score on Friday evening, the sleep duration from Tuesday night (duration day before yesterday's sleep) and Wednesday night (duration yesterday's sleep) were included as features, in addition to the sleep duration from Thursday night (duration today's sleep). From this, we see that having about 4 to 6 hours of sleep the previous day (Wednesday night) was predictive of today's (Friday's) perceived stress and sad mood.

Academic activity. The model selected low study duration (0-2 hours) as a high predictor of calmness. In contrast, high study duration of more than 8 hours was the strongest predictor of stress and perceived sickness. We also see spending more than 8 hours on campus as a high predictor of stress. We do not know if the students were studying while on campus, but given the amount of time spent, it is highly likely they were studying. ${ }^{5}$

Physical activity. Having 1 to 2 hours of exercise a day was found to be a high predictor of calmness within the study participants. This is in line with several research studies that have shown that physical activity improves well-being $[11,16,17,32]$. In contrast, we also found that not having any exercise was a high predictor of stress, sad mood, and sickness.

Caffeine and alcohol consumption. Caffeine and alcohol consumption had opposite effects on the prediction of calmness. Although caffeine consumption was found to be a predictor of high stress, alcohol was a predictor of calmness. We note that the students typically fill out the surveys after 8PM, and we do not have information on the amount of alcohol consumed by the students. Therefore, there are two possible explanations for the reason alcohol shows up as a predictor of calm. First, we may be seeing the calming effect of alcohol if the evening surveys were filled out soon after taking alcohol. Second, in the dataset, those who reported alcohol consumption had a $65 \%$ chance of doing so on the weekend (Friday-Sunday), and the weekend has been shown to be associated with better psychological well-being [23]. So we may be seeing the weekend effect mixed in with the alcohol effect.

In summary, we have seen that given the normalized bag-of-behaviors features, LASSO was able to select intuitively correct features to be predictors of self-reported stress, mood, and health. It is important to note that

\footnotetext{
${ }^{5} \mathrm{~A}$ possible confounder is that some students in the study lived in a couple of dormitories that were part of the geographical boundaries we defined as being "on campus."
} 


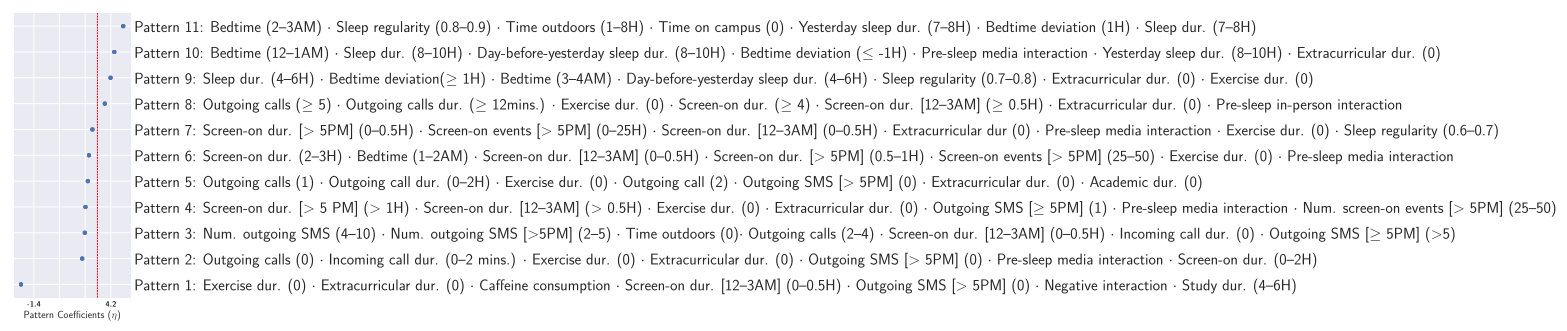

Fig. 11. An 11-pattern sLDA model fit to the SNAPSHOT data for self-reported stress (the red vertical line represents mean of the positive coefficients). Pattern coefficient decrease from top to bottom. In each pattern, the behaviors are ordered from left to right starting from the most probable behavior.

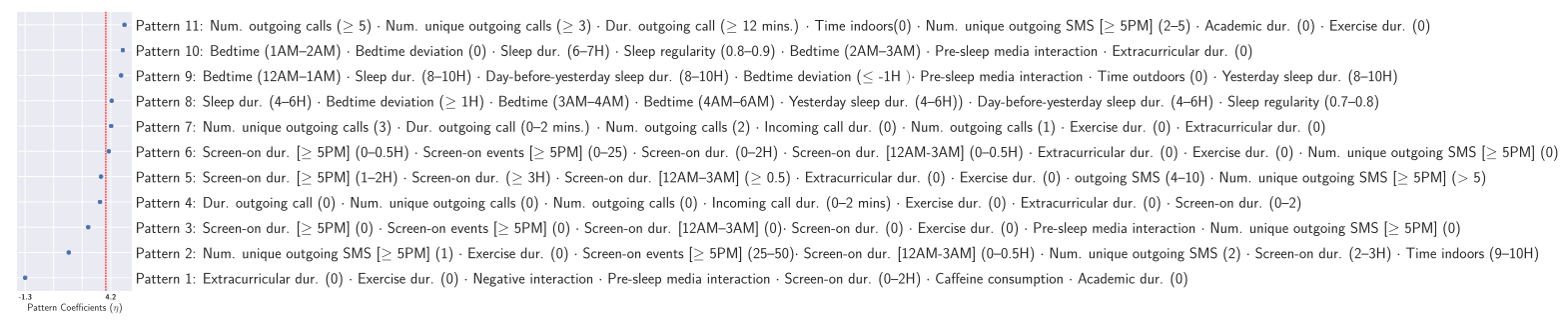

Fig. 12. An 11-pattern sLDA model fit to the SNAPSHOT data for self-reported mood (the red vertical line represents mean of the positive coefficients). Pattern coefficient decrease from top to bottom. In each pattern, the behaviors are ordered from left to right starting from the most probable behavior.

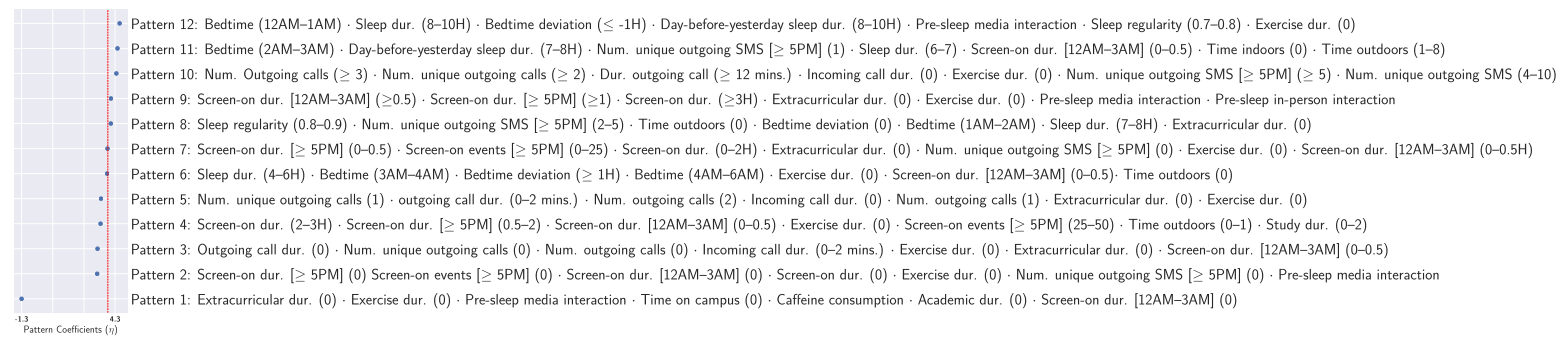

Fig. 13. A 12-pattern sLDA model fit to the SNAPSHOT data for self-reported health (the red vertical line represents mean of the positive coefficients). Pattern coefficients decrease from top to bottom. In each pattern, the behaviors are ordered from left to right starting from the most probable behavior.

the relationships discovered are associative. As a result, further studies will be required to understand causal relationships.

\subsection{SLDA}

The sLDA model learned latent patterns that are meaningful representations of health behavior. Here, we further analyze these patterns. Since we are interested in good predictive patterns, we select $K=11$ for self-reported stress and mood and $K=12$ for self-reported health for our discussion because it provides us with the highest binary prediction accuracy (when the threshold is set to 50 for self-reported stress and set to mean for selfreported mood and health). Figures 11, 12, and 13 show the sLDA model fit to our data. For each learned pattern, we highlight the seven health behaviors with the highest probability of occurrence. 


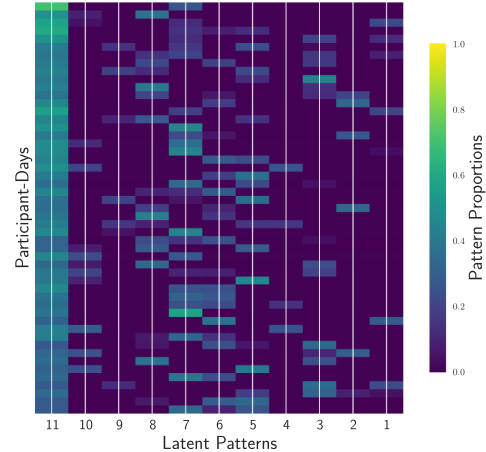

(a) High calm

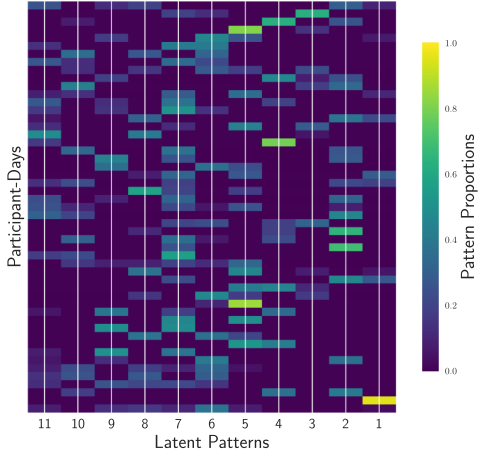

(b) Mid stressed-calm

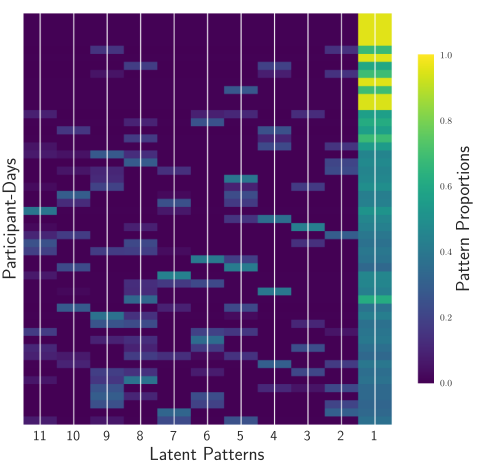

(c) High stressed

Fig. 14. Learned day-pattern proportions $(\theta)$ in three different levels of the self-reported stress label $(y)$ : 50 participant-days with $y \geq 80$ (a), 50 participant-days with $55 \leq y<65$ (b), and 50 participant-days with $y \leq 40$ (c). Pattern 11 is the most calm pattern, and pattern 1 is the most stressed pattern.

For self-reported stress, the pattern with the most negative coefficient pattern 1 (bottom) comprises highly probable behaviors such as no exercise, caffeine consumption, very little social interaction, study duration of 4 to 6 hours, and negative social interaction. The pattern with the most positive coefficient pattern 11 (top) consists of highly probable behaviors such as high SR, going to bed around the average bedtime of the study population, spending time outdoors, sufficient sleep the night before, and very little phone usage between the hours of midnight and 3AM (likely because the participant is asleep). We also see SR increasing as the patterns become more positively associated with calmness, from $S R=0.6$ to 0.7 in pattern 7 to $S R=0.8$ to 0.9 in pattern 11 . The patterns in between have a mix of health behaviors, which provide evidence that the "positive" behaviors start to compensate for the others as the patterns become more positively correlated with calmness. For example, in pattern 9, we see a high SR of 0.7 to 0.8 but late bedtime, 3-4AM, and low sleep duration for two consecutive days, 4 to 6 hours. It may be that the high SR helps build resilience, enabling an individual to weather a couple days of late bedtime and short sleep without the expected increase in stress.

In Figure 12, the pattern most positively associated with good mood, pattern 11 (top), is made up of behaviors that indicate social interaction via the cell phones. In patterns 9 and 10, we also have behaviors like high SR, bedtime between the hours of $12 \mathrm{AM}$ and 2AM, and 6 to 10 hours of sleep being highly associated with high happy mood. However, pattern 1 (bottom), the pattern most associated with sad mood, consists of highly probably behaviors such as negative social interaction and no exercise. For self-reported health (shown in Figure 13), pattern 12 (top), which has the most positive coefficient, consists of highly probably good sleep behaviors, such as high SR ( $\mathrm{SR}=0.7-0.8)$; 8 to 10 hours of sleep; and, relative to the study cohort, an early bedtime of $12 \mathrm{AM}$ to $1 \mathrm{AM}$. This is in line with previous research that found that regular sleep is essential for good health and well-being [25].

These findings are consistent with our belief that complex combinations of health behaviors lead to different outcomes, and models that only learn prediction rules or that focus on only analysis of the impact of single behaviors are insufficient to uncover these complex latent patterns that drive real-world data.

One advantage that sLDA has over LASSO is that it learns the day-pattern probability distributions over each participant's day. This gives us insight into how the patterns expressed in each person's day contribute to the self-reported perceived stress. In addition, this personalized distribution over patterns illustrates how the sLDA model is able to capture the similarity in the behaviors of participants with comparable self-reported stress, mood, or health. Figures 14, 15, and 16 show the pattern proportions learned in 50 different participants randomly chosen from low, mid, and high self-reported stress, mood, and health levels, respectively. Participants who reported high well-being measures have the most positive pattern dominantly expressed; participants with 


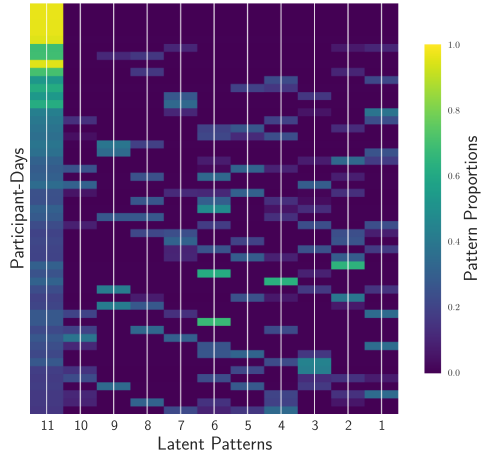

(a) High happy mood

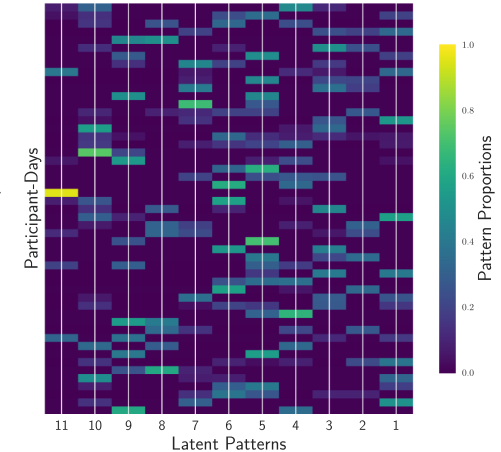

(b) Mid sad-happy

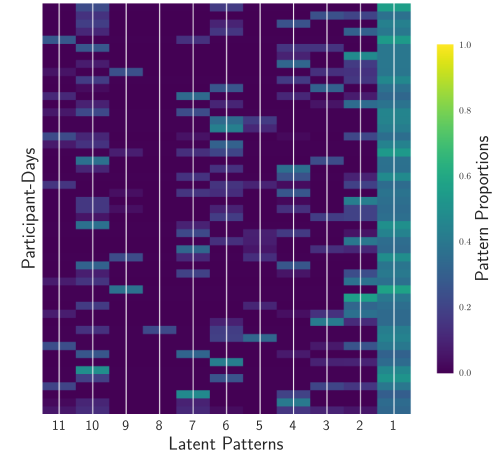

(c) High sad mood

Fig. 15. Learned day-pattern proportions $(\theta)$ in three different levels of the self-reported mood label $(y)$ : 50 participant-days with $y \geq 80$ (a), 50 participant-days with $55 \leq y<65$ (b), and 50 participant-days with $y \leq 40$ (c). Pattern 11 is the most happy pattern, and pattern 1 is the most sad pattern.

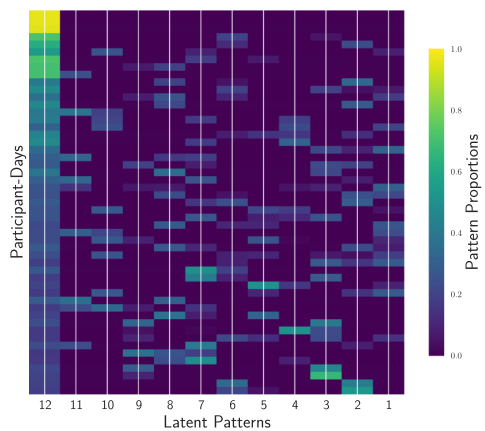

(a) High healthy

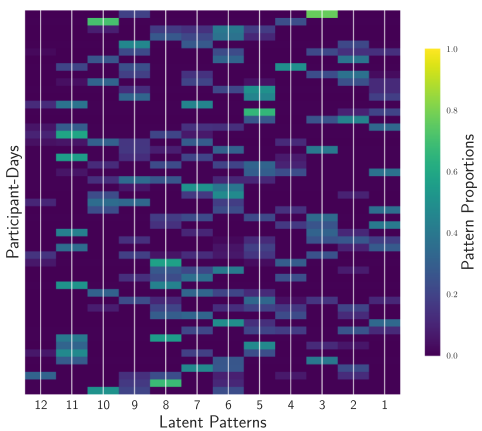

(b) Mid sick-healthy

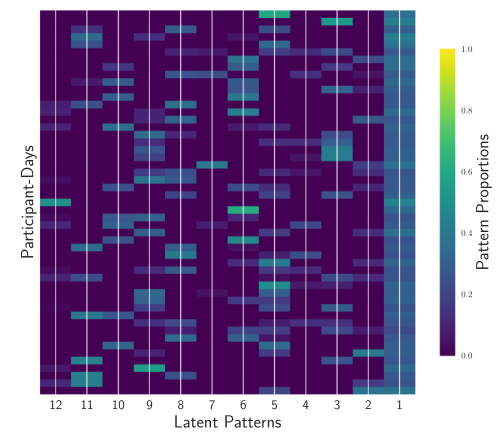

(c) High sick

Fig. 16. Learned day-pattern proportions $(\theta)$ in three different levels of the self-reported health label $(y)$ : 50 participant-days with $y \geq 80$ (a), 50 participant-days with $55 \leq y<65$ (b), and 50 participant-days with $y \leq 40$ (c). Pattern 12 is the most healthy pattern, and pattern 1 is the most sick pattern.

low self-reported well-being measures have the most negative pattern dominantly expressed; and participants in the mid-range of 55 to 65 self-reported well-being measures have varying representations of patterns expressed in each day.

\subsection{Case Study}

In this section, we use two case studies to illustrate how we can provide evidence-based insights using the latent patterns learned by the sLDA and the JS distance.

Case 1. Participant $A$ is on the evening of the 18th day of the study. Figure 17 shows the self-reported calm scores of the participant for the past 17 days. Figures 18, 19, and 20 show box plots of the behaviors exhibited by participant A during the past 17 days.

The health behaviors are ordered according to the average of the self-reported calm scores on the days the behaviors are exhibited. The behaviors exhibited during Day 18 are shown in Table 3. Given these behaviors, the sLDA model predicted that the participant will be in the mid-high calm range in the evening of Day 18 . The true score reported by the participant later that evening is 75 . 


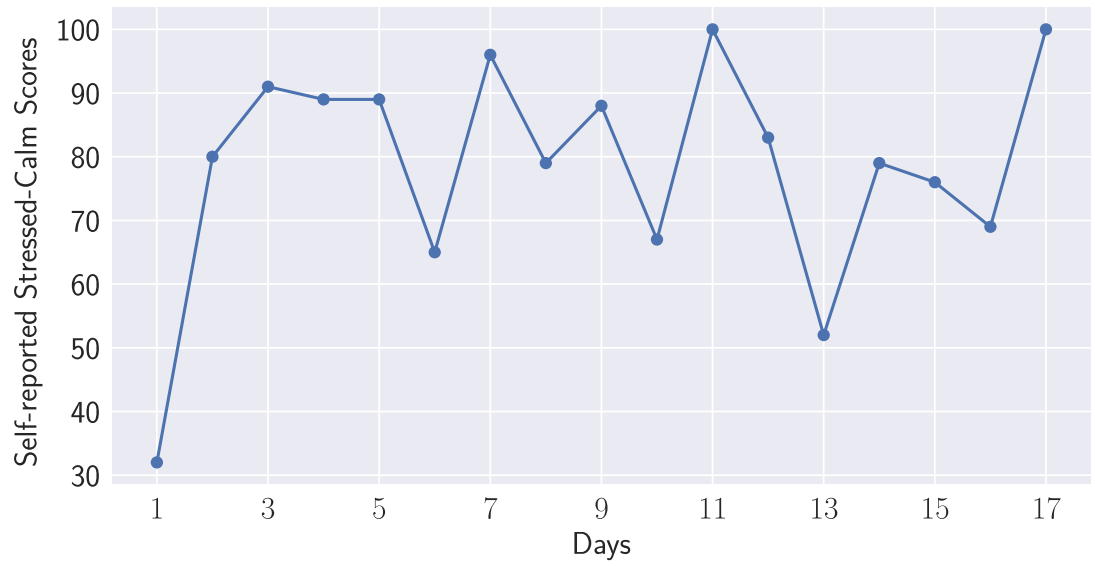

Fig. 17. Self-reported stressed-calm scores of Participant A for the past 17 days of the study.

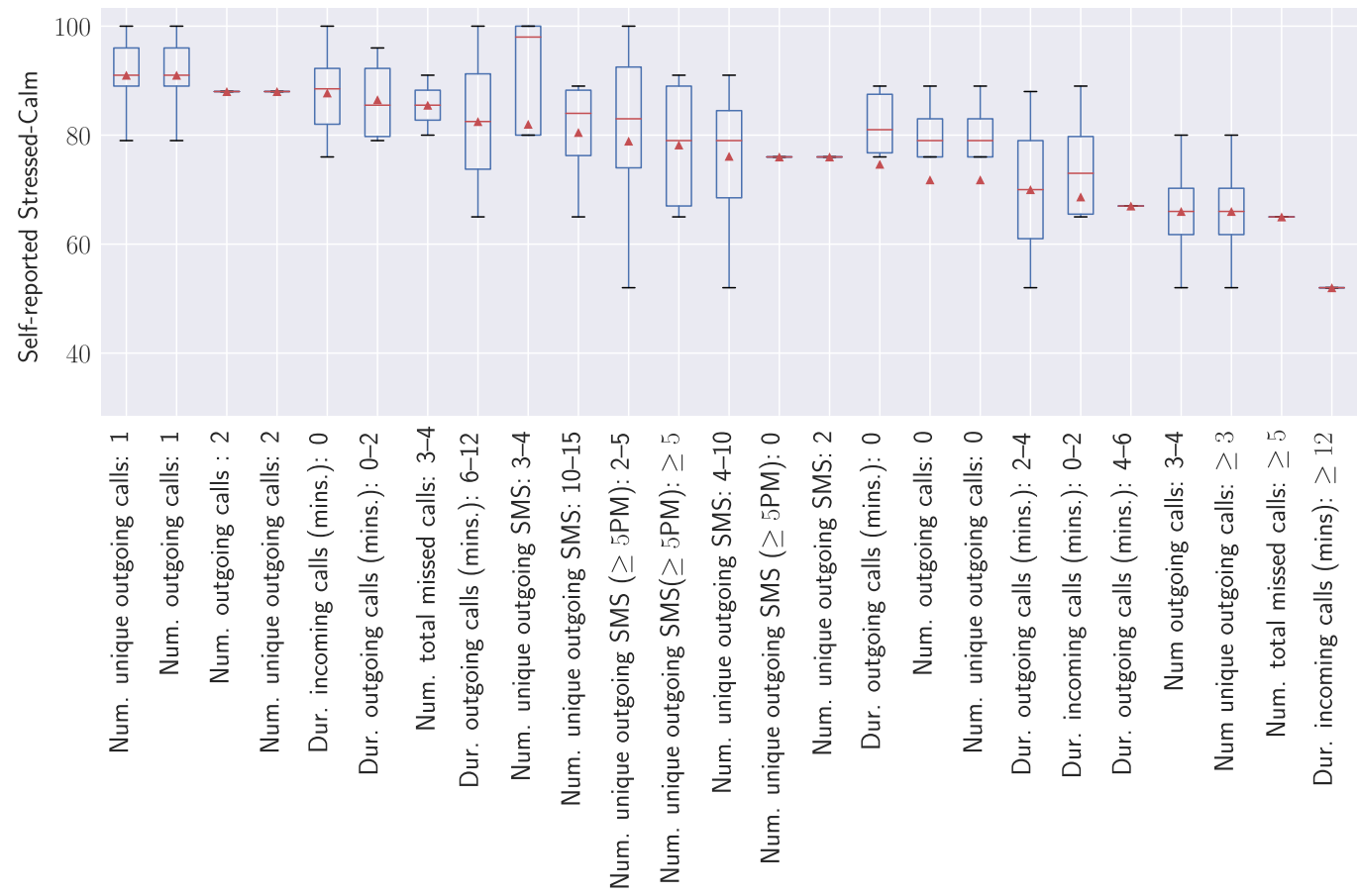

Fig. 18. Health behaviors extracted from cell phone usage of Participant A for the past 17 days, ordered by the average of self-reported stressed-calm scores on days the behavior was present $(0=$ most stress; $100=$ most calm).

We query the day-pattern distributions used to train the model and report the two most similar participantdays with calm scores higher than the predicted calm scores for Participant A in Table 3.

The two participants returned in the query had similar sleep duration the night before, but they went to bed earlier. Similar Participant 2 had better sleep duration three nights in a row, and on the previous night, bedtime was the same as the average bedtime during the study (no deviation). Based on the health behaviors of the similar participants, two recommendations to Participant A could be as follows: Go to bed an hour or two earlier, and 


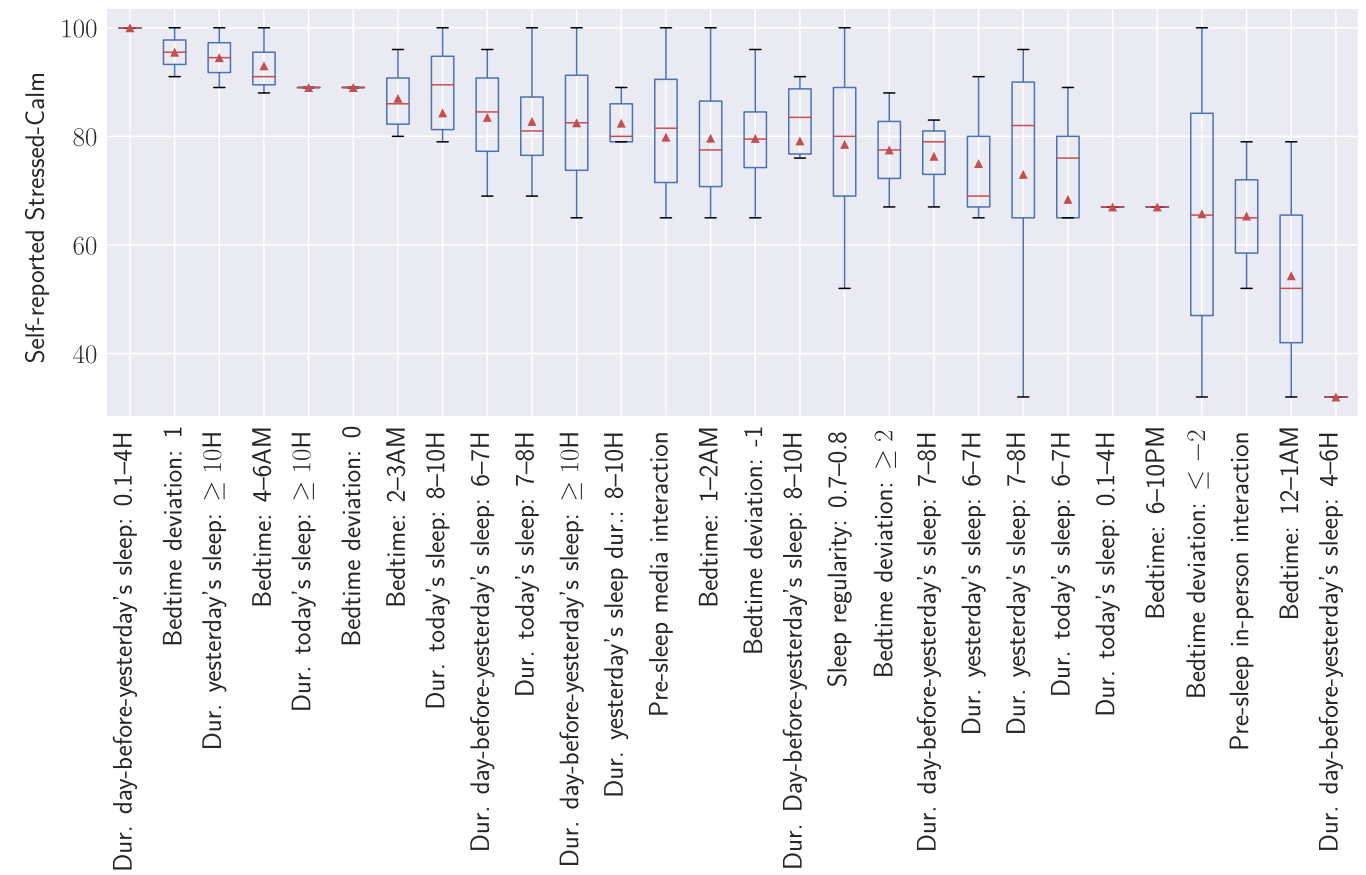

Fig. 19. Health behaviors corresponding to sleep extracted from Actiwatch of Participant A for the past 17 days, ordered by the average of self-reported stressed-calm scores on days the behavior was present $(0=$ most stress; $100=$ most calm $)$.

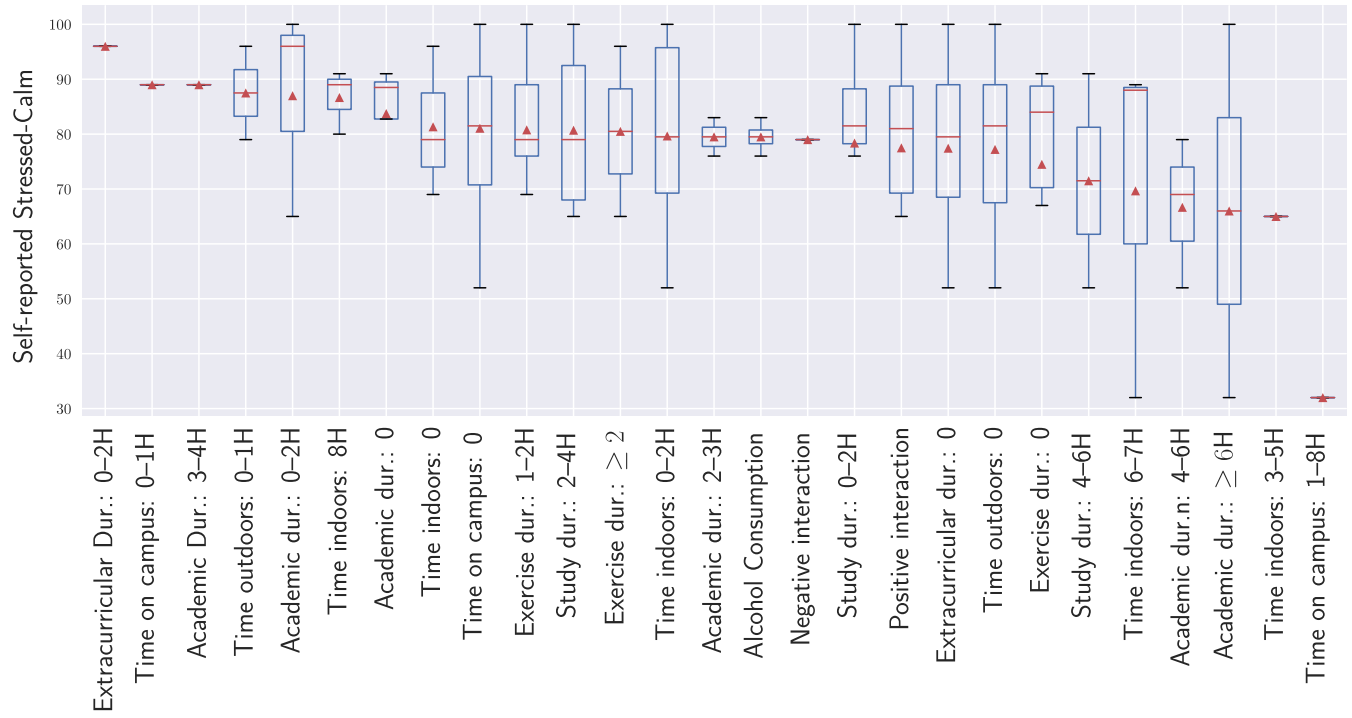

Fig. 20. Health behaviors extracted from GPS coordinates and twice daily surveys of Participant A for the past 17 days, ordered by the average of self-reported stressed-calm scores on days the behavior was present $(0=$ most stress; $100=$ most calm). 
Table 3. Health-Related Behaviors of Participant A (Exhibited on Day 18) and the Two Participants Most Similar to Participant A

\begin{tabular}{|c|c|c|c|c|}
\hline Modality & Behaviors & $\begin{array}{c}\text { Participant } A \\
\text { True Calm Score }=75\end{array}$ & $\begin{array}{c}\text { Similar Participant } 1 \\
\text { Calm Score }=87\end{array}$ & $\begin{array}{c}\text { Similar Participant } 2 \\
\text { Calm Score }=91\end{array}$ \\
\hline \multirow{10}{*}{ Cell phone usage } & Num. total outgoing calls & 2 & $\geq 5$ & $3-4$ \\
\hline & Num. unique outgoing calls & 2 & $\geq 3$ & $\geq 3$ \\
\hline & Num. on/off events (5PM-midnight) & - & $25-50$ & $25-50$ \\
\hline & Num. unique outgoing SMS (5PM-midnight) & 0 & $2-5$ & $2-5$ \\
\hline & Num. unique outgoing SMS (all day) & 1 & $4-10$ & $4-10$ \\
\hline & Duration incoming calls (all day) (min.) & 0 & $\geq 12 \mathrm{~min}$ & 0 \\
\hline & Duration outgoing calls (all day) (min.) & $0-2 \mathrm{~min}$. & $\geq 12 \mathrm{~min}$ & $\geq 12 \mathrm{~min}$ \\
\hline & Duration on/off events (all day) (hours) & - & $2-3$ hours & $2-3$ hours \\
\hline & Duration on/off events (midnight-3AM) (hours) & - & 0.5 hours & $0-0.5$ hours \\
\hline & Duration on/off events (5PM-midnight) (hours) & - & $0.5-1$ hours & $0.5-1$ hours \\
\hline \multirow{6}{*}{ Actiwatch } & Duration today’s sleep (hours) & $6-7$ hours & $6-7$ hours & $6-7$ hours \\
\hline & Bedtime (hour of the day) & $4 \mathrm{AM}-6 \mathrm{AM}$ & $3 \mathrm{AM}-4 \mathrm{AM}$ & $1 \mathrm{AM}-2 \mathrm{AM}$ \\
\hline & Duration yesterday's sleep (hours) & 6-7 hours & $7-8$ hours & $7-8$ hours \\
\hline & Duration day before yesterday's sleep (hours) & $7-8$ hours & $4-6$ hours & $8-10$ hours \\
\hline & Bedtime deviation from participant's mean (hours) & 1 hour & 1 hour & 0 hour \\
\hline & Weekly SR & $0.7-0.8$ & $0.8-0.9$ & $0.7-0.8$ \\
\hline \multirow{3}{*}{ Location } & Time on campus (hours) & 0 & 0 & 0 \\
\hline & Time indoors (hours) & $0-2$ hours & $6-7$ hours & 0 \\
\hline & Time outdoors (hours) & 0 & $1-8$ hours & 0 \\
\hline \multirow{7}{*}{ Behavioral surveys } & Study duration (hours) & $2-4$ hours & 0 & 0 \\
\hline & Exercise duration (hours) & 0 & 0 & 0 \\
\hline & Extracurricular duration (hours) & 0 & 0 & 0 \\
\hline & Academic duration (hours) & 0 & $2-3$ hours & $4-6$ hours \\
\hline & Type of social interaction & Positive & Positive & Positive \\
\hline & Pre-sleep interaction & Media & In-person & In-person and media \\
\hline & Alcohol or caffeine consumption & Alcohol & None & Caffeine \\
\hline
\end{tabular}

Bold entries highlight possible recommendations.

also have more social interactions the next day. From Figure 19, we see that the average calm scores over the past days Participant A went to bed at 2AM to $3 \mathrm{AM}$ and $1 \mathrm{AM}$ to $2 \mathrm{AM}$ were 87 and 80 , respectively. Additionally in Figure 18, Participant A had an average reported score of 82.5 over the days when outgoing call duration was between 6 and 12 minutes. Therefore, these recommendations are reasonable and might improve the well-being of Participant A.

Case 2. Participant B is on the 14th day of the study. Figure 21 shows the self-reported stressed-calm scores of the participant for the past 13 days. We can see that the participant reported high stress (calm score < 55) for most of the days. Figures 22, 23, and 24 show box plots of the past behaviors exhibited by Participant B. The behaviors are ordered according to the average self-reported calm scores.

The behaviors exhibited on Day 14 are shown in Table 4. Given these behaviors, the sLDA model predicted that the participant is in the high stress range (self-report calm scores $<50$ ). The actual score reported by the participant is 21 . We query the day-pattern distributions used to train the model and report the two most similar participant-days with calm scores higher than the predicted calm scores for Participant B in Table 4. 


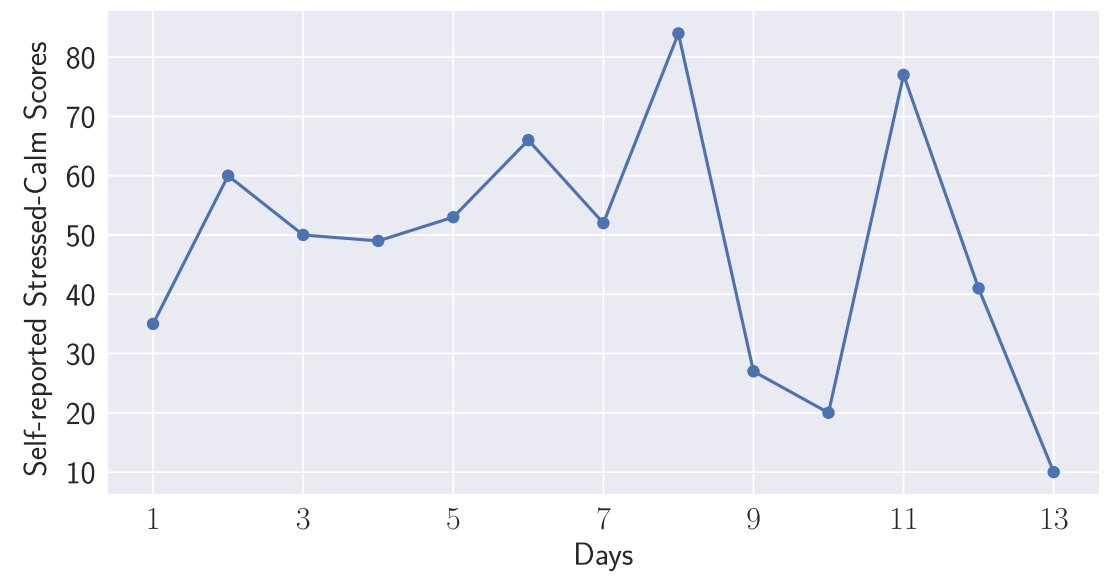

Fig. 21. Self-reported stressed-calm scores of Participant B for the past 13 days of the study.

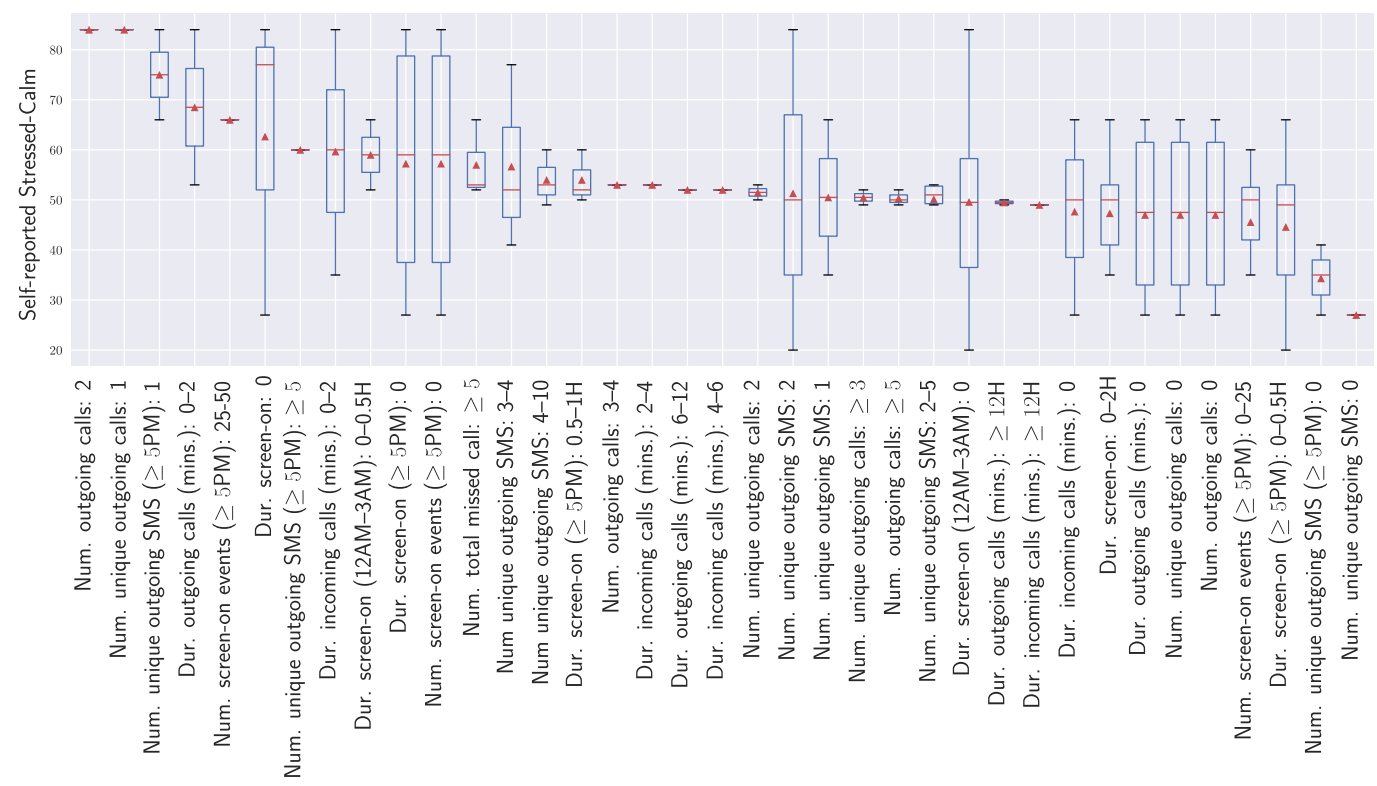

Fig. 22. Health behaviors extracted from cell phone usage of Participant B for the past 13 days, ordered by the average of self-reported stressed-calm scores on days the behavior was present $(0=$ most stress; $100=$ most calm).

Health behaviors such as less than 4 hours of sleep, minimum phone usage, negative social interactions, and late bedtime the night before are present in Participant B's high stress day. However, the two similar participants returned by the model have very high calm scores and exhibit behaviors such as moderate phone usage, only positive interactions, adequate sleep and going to bed earlier than their average bedtime. To improve well-being, it could be recommended that Participant B go to bed an hour earlier, sleep for at least 6 hours that night, and keep a regular bedtime going forward. In addition, avoiding negative interactions or seeking positive interactions or both could help improve the well-being of Participant B. Figure 23 shows higher average calm scores of 85 and 67 on days Participant B went to bed between 2AM and 3 AM and slept for more than 8 hours, respectively. 


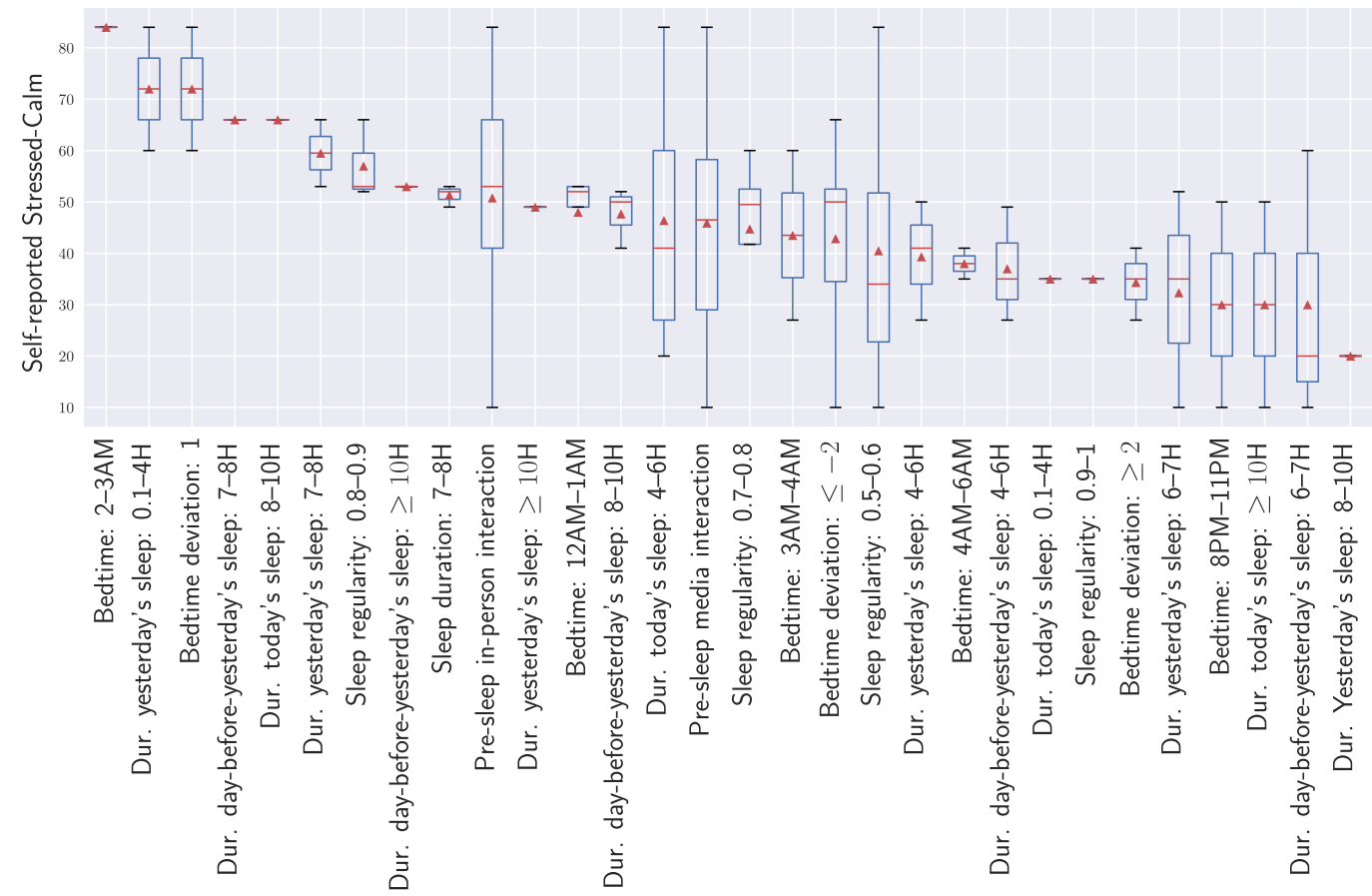

Fig. 23. Health behaviors corresponding to sleep extracted from Actiwatch of Participant B for the past 13 days, ordered by the average of self-reported stressed-calm scores on days the behavior was present $(0=$ most stress; $100=$ most calm).

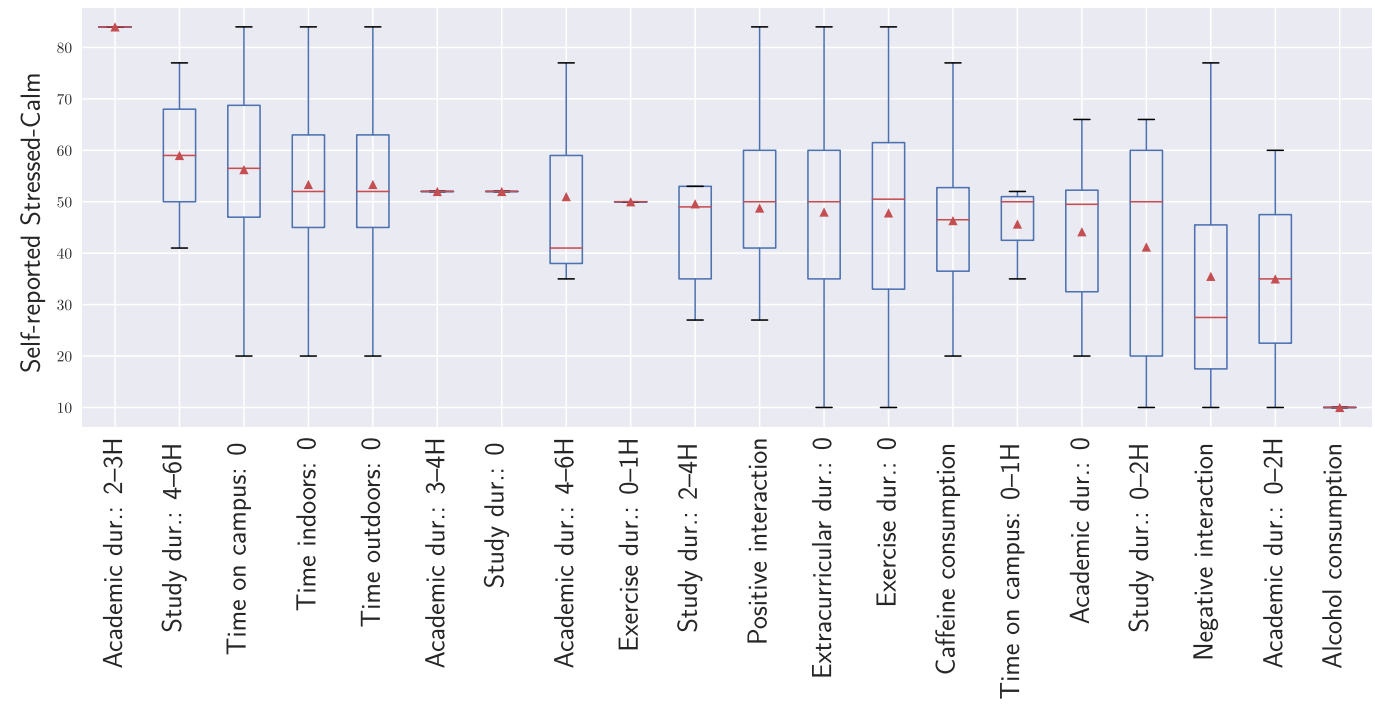

Fig. 24. Health behaviors extracted from GPS coordinates and twice daily surveys of Participant B for the past 13 days, ordered by the average of self-reported stressed-calm scores on days the behavior was present $(0=$ most stress; $100=$ most calm). 
Table 4. Health-Related Behaviors of Participant B (Exhibited on Day 14) and the Two Participants Most Similar to Participant B

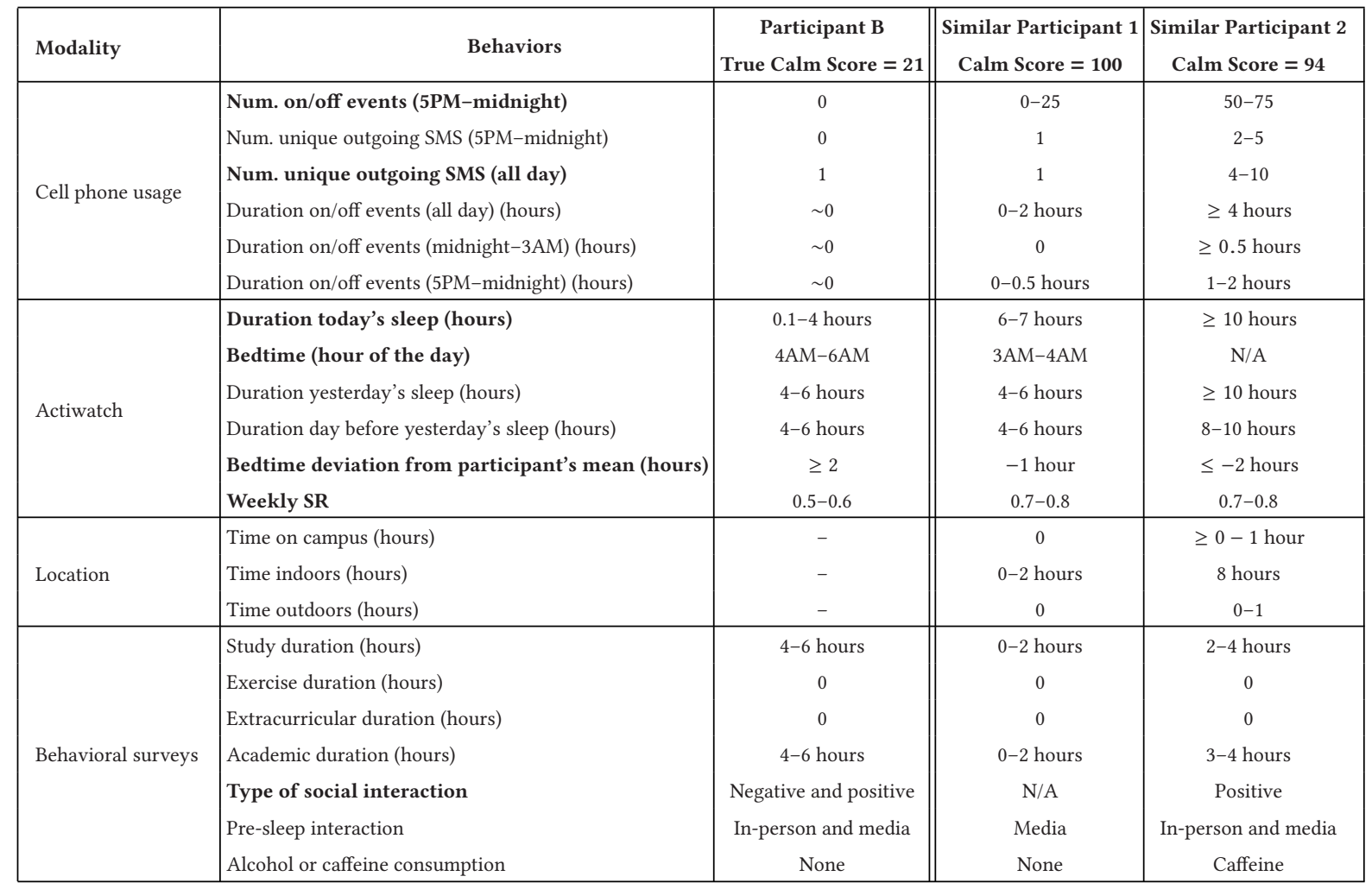

Bold entries highlight possible recommendations.

In Figure 24, we see an average score of 50, with a maximum calmness score of 85 on the days Participant B had positive social interactions. These recommendations could help improve Participant B’s well-being.

\section{CONCLUSION AND FUTURE WORK}

We proposed a novel framework to map multi-modal data collected in the "wild" to meaningful representations of health behavior, which are then used with sLDA to learn patterns of modifiable health behaviors predictive of stress, a major factor in well-being as well as of sad-happy mood and overall physical state of sick-healthy. We compared the predictive performance of the patterns learned with sLDA to the patterns learned with an unsupervised LDA, showing that the sLDA significantly outperforms LDA in binary prediction of stress, mood, and physical health. We additionally compared the performance of sLDA to LASSO and found that the sLDA outperforms LASSO in binary binary prediction of stress, mood, and physical health.

We analyzed the latent structure uncovered by the sLDA model and showed how specific patterns of behavior are associated with self-reported sad-happy mood, stressed-calm state, and sick-healthy state. Previous work has shown correlations with single classes of behaviors such as modifying sleep or social interaction [25, 30], but we have shown how combinations of specific behaviors, such as going to bed 2 hours earlier or later than usual, getting 7 to 8 versus 4 to 6 hours of sleep, and having positive versus negative social interactions, are associated with student well-being measures. We can only speculate about cause and effect in these findings, and in many of them we may expect causality to go in both directions; for example, people who have poor well-being exercise 
less, but also not exercising may contribute to poor well-being. Through this work, we have shown that the sLDA model finds informative structure, which produces patterns that are predictive of well-being, and connects with an intuitive understanding of health behaviors.

In addition, we have illustrated how the sLDA-based personalized recommendations can be applied in real life, using case studies of two participants in the SNAPSHOT study: one with a mid-high calm score and another with a low calm score

At the same time, this work has limitations that can be improved in future work. First, the sLDA model did not consider the inherent temporal effects of behaviors on well-being. Although each day is modeled independently, we included information about the previous 2 days of sleep to test the hypothesis that sleep features of past days affect today's well-being. We found two instances supporting this hypothesis: first, in the LASSO model, sleeping 4 to 6 hours prior to the previous day was predictive of high stress and sad mood; second, in the sLDA model, the most calm pattern had day-before-yesterday sleep duration of 7 to 8 hours as a highly probably behavior, the next calm predictive pattern found going to bed 1 or more than 2 hours earlier than your personal average bedtime (bedtime deviation -1 or $\leq-2$, respectively) as a highly probable behavior, and the most healthy pattern had daybefore-yesterday sleep duration of 8 to 10 hours as being predictive of good health. With the collection of more data, we can use a supervised variant of dynamic topic models [2] to learn how time-varying behaviors affect an individual's well-being. Second, our work used self-reported labels as a proxy for well-being measurement. However, these labels can be inconsistent across and within users. Our team has an ongoing effort to produce objective labels measuring physiological stress from SNAPSHOT data- work that is outside the scope of this article.

A third limitation is the model's predictive accuracy. Using data from the SNAPSHOT study, other models have been able to provide more accurate predictions. In Taylor et al. [29], for example, the Bayesian multi-task learning model and personalized neural network framework achieved higher prediction accuracies. One reason for their higher prediction performance is that other features in addition to modifiable behaviors were used to learn the prediction rule (e.g., they used physiology). This gave the models more information to distinguish between low or high stress, mood, or health days. In contrast, this work restricted features to modifiable behaviors, like bedtime and screen time, yielding variables that individuals can change behaviorally for future causality studies. Nonetheless, our results showed that modifiable behaviors have a significant predictive association with a participant's mental health and well-being. In addition, the Taylor et al. problem was made easier than ours by discarding data corresponding to the middle $20 \%$ of the self-reported well-being labels, throwing out ambiguous "middle" values. For the work in this article, we did not throw out the middle $20 \%$ because sLDA uses a generalized linear regression framework to model the real values from 0 to 100 that were originally reported. This is valuable because we can directly predict a finer estimate of self-reported well-being rather than just a binary category. For example, individuals might be interested in seeing the trends in their self-reported stress, mood, or health and whether or not it is gradually or suddenly increasing. Algorithms that only predict oscillating values between high and low well-being measures will not be as useful. We converted the real-valued predictions to binary labels only for the purposes of simplifying model comparisons and showed the gains were robust using two different thresholds.

Fourth, a main limitation is that the evidence-based recommendations are currently based on statistical associations and not on behaviors that have been proven to have causal influence. We believe that these recommendations are, however, useful ones to test for subsequent causal experiment designs. Further, it is unclear how much the advice based on others will be valuable for somebody using only a measure of similar behaviors. With a larger dataset, and with feedback as to whether taking the advice (actually carrying out the recommended behaviors) leads to the expected improved (or not) well-being scores, it will be possible to learn not only better recommendations but also better ways to combine information from "similar" people and similar contexts to improve the recommendations. 
To get closer to the goal of building a real-world behavior recommendation system, future work can expand this approach in the following directions.

Longer study. We used data from a 30-day study to train the model. Recruiting more people for longer studies up to a semester, for example, will provide more data that can enable the learning of better representations of health behaviors and thus make better personalized recommendations. We note that a pilot semester-long study was run on 15 people, so a semester-long study is feasible.

Integrated development environments. Developing integrated development environments will make it easier to collect data from participants, run the model, and make recommendations in real time. In addition, the integrated development environment will enable researchers to study the effectiveness of the recommendations by also collecting data after the participants have followed the recommendations.

Data analysis and follow-up study. It is important to test the validity or effectiveness of the recommendations (i.e., whether or not well-being was improved) and to include any feedback into the model in an iterative process. Analyzing the data collected after recommendations are followed will provide us with valuable insight. A followup study that can test the causal relationships between some of the health behaviors recommended and wellbeing will also strengthen the health behavior recommendations.

In conclusion, the new modeling framework we present can uncover latent patterns of behaviors that are most predictive of a participant's well-being as measured by self-reported stress, mood, and health, and provide evidence-based insights to individuals seeking to improve their well-being. Although we use data from a student population, we believe that given any set of modifiable behaviors, our framework can be applied to other population groups.

\section{ACKNOWLEDGMENTS}

We would like to thank Dr. Akane Sano, Dr. Charles Czeisler, Dr. Elizabeth Klerman, Sara Taylor, Natasha Jaques, Craig Ferguson, and other SNAPSHOT project members (https://snapshot.media.mit.edu/team) for their help in designing and running the SNAPSHOT study. In addition, we would like to thank Hanna Wallach for the initial discussions at NeurIPS that led to this work.

\section{REFERENCES}

[1] Yoshua Bengio, Aaron Courville, and Pascal Vincent. 2013. Representation learning: A review and new perspectives. IEEE Transactions on Pattern Analysis and Machine Intelligence 35, 8 (2013), 1798-1828.

[2] David M. Blei and John D. Lafferty. 2006. Dynamic topic models. In Proceedings of the 23rd International Conference on Machine Learning. ACM, New York, NY, 113-120.

[3] David M. Blei and Jon D. McAuliffe. 2007. Supervised topic models. In Advances in Neural Information Processing Systems.

[4] David M. Blei, Andrew Y. Ng, and Michael I. Jordan. 2003. Latent Dirichlet allocation. Journal of Machine Learning Research 3 (2003), 993-1022.

[5] Taridzo Chomutare, Eirik Arsand, and Gunnar Hartvigsen. 2011. Mobile peer support in diabetes. Studies in Health Technology and Informatics 169 (2011), 48-52. http://www.ncbi.nlm.nih.gov/pubmed/21893712.

[6] Ed Diener and Martin Seligman. 2017. Beyond money: Progress on an economy of well-being. Psychological Science in the Public Interest 5, 1 (2017), 1-31.

[7] Katayoun Farrahi and Daniel Gatica-Perez. 2014. A probabilistic approach to mining mobile phone data sequences. Personal and Ubiquitous Computing 18, 1 (2014), 223-238.

[8] Robert G. Farrell, Catalina M. Danis, Sreeram Ramakrishnan, and Wendy A. Kellogg. 2012. Intrapersonal retrospective recommendation: Lifestyle change recommendations using stable patterns of personal behavior. In Proceedings of the ACM RecSys 1st International Workshop on Recommendation Technologies for Lifestyle Change, Vol. 891. 24-28. DOI : https://doi.org/10.1145/2365952.2366045

[9] Laura Ferrari and Marco Mamei. 2011. Discovering daily routines from Google Latitude with topic models. In Proceedings of the IEEE International Conference on Pervasive Computing and Communications Workshops. 432-437.

[10] Mouzhi Ge, Mehdi Elahi, Ignacio Fernaández-Tobías, Francesco Ricci, and David Massimo. 2015. Using tags and latent factors in a food recommender system. In Proceedings of the 5th ACM International Conference on Digital Health. 105-112. DOI : https://doi.org/10.1145/ 2750511.2750528 
[11] Suzanne Hudd, Jennifer Dumlao, Diane Erdmann-Sager, Daniel Murray, Emily Phan, Nicholas Soukas, and Nori Yokozuka. 2000. Stress at college: Effects on health habits, health status and self-esteem. College Student fournal 34, 2 (2000), 217-227.

[12] Tâm Huynh, Mario Fritz, and Bernt Schiele. 2008. Discovery of activity patterns using topic models. In Proceedings of the 10th ACM International Conference on Ubiquitous Computing. 10-19.

[13] Imran Shuja Khawaja, Joseph J. Westermeyer, Prashant Gajwani, and Robert E. Feinstein. 2009. Depression and coronary artery disease: The association, mechanisms, and therapeutic implications. Psychiatry (Edgmont) 6, 1 (2009), 38.

[14] Jia Li, Yu Rong, Helen Meng, Zhihui Lu, Timothy Kwok, and Hong Cheng. 2018. TATC: Predicting Alzheimer's disease with actigraphy data. In Proceedings of the 24th ACM SIGKDD International Conference on Knowledge Discovery and Data Mining. ACM, New York, NY, 509-518.

[15] Ehimwenma Nosakhare and Rosalind Picard. 2019. Probabilistic latent variable modeling for assessing behavioral influences on wellbeing. In Proceedings of the 25th ACM SIGKDD International Conference on Knowledge Discovery and Data Mining. ACM, New York, NY, 2718-2726.

[16] Gregory A. Panza, Beth A. Taylor, Paul D. Thompson, C. Michael White, and Linda S. Pescatello. 2017. Physical activity intensity and subjective well-being in healthy adults. fournal of Health Psychology 24, 9 (2017), 135910531769158.

[17] Tim Pawlowski, Paul Downward, and Simona Rasciute. 2011. Subjective well-being in European countries-On the age-specific impact of physical activity. European Review of Aging and Physical Activity 8, 2 (2011), 93-102.

[18] Santi Phithakkitnukoon, Teerayut Horanont, Giusy Di Lorenzo, Ryosuke Shibasaki, and Carlo Ratti. 2010. Activity-aware map: Identifying human daily activity pattern using mobile phone data. In Proceedings of the International Workshop on Human Behavior Understanding. 14-25.

[19] Dinh Phung, Brett Adams, Kha Tran, Svetha Venkatesh, and Mohan Kumar. 2009. High accuracy context recovery using clustering mechanisms. In Proceedings of the IEEE International Conference on Pervasive Computing and Communications. 1-9.

[20] Frans Pouwer, Nina Kupper, and Marcel C. Adriaanse. 2010. Does emotional stress cause type 2 diabetes mellitus? A review from the European Depression in Diabetes (EDID) research consortium. Discovery Medicine 9, 45 (2010), 112-118.

[21] Tom Quisel, Luca Foschini, Alessio Signorini, and David C. Kale. 2017. Collecting and analyzing millions of mHealth data streams. In Proceedings of the 23rd ACM SIGKDD International Conference on Knowledge Discovery and Data Mining. ACM, New York, NY, 19711980.

[22] Mashfiqui Rabbi, Min Hane Aung, Mi Zhang, and Tanzeem Choudhury. 2015. MyBehavior: Automatic personalized health feedback from user behaviors and preferences using smartphones. In Proceedings of the ACM International foint Conference on Pervasive and Ubiquitous Computing. 707-718. DOI : https://doi.org/10.1145/2750858.2805840

[23] Richard M. Ryan, Jessey H. Bernstein, and Kirk Warren Brown. 2010. Weekends, work, and well-being: Psychological need satisfactions and day of the week effects on mood, vitality, and physical symptoms. Journal of Social and Clinical Psychology 29, 1 (2010), 95-122.

[24] Akane Sano. 2015. Measuring College Students' Sleep, Stress and Mental Health with Wearable Sensors and Mobile Phones. Ph.D. Dissertation. MIT.

[25] Akane Sano, Andrew J. Phillips, Andrew W. McHill, Sara Taylor, Laura K. Barger, Charles A. Czeisler, and Rosalind W. Picard. 2017. Influence of weekly sleep regularity on self-reported wellbeing. Sleep 40, Suppl. 1 (2017), A67-A68.

[26] Akane Sano, Sara Taylor, Andrew W. McHill, Andrew J. Phillips, Laura K. Barger, Elizabeth Klerman, and Rosalind Picard. 2018. Identifying objective physiological markers and modifiable behaviors for self-reported stress and mental health status using wearable sensors and mobile phones. fournal of Medical Internet Research 20, 6 (2018), e210.

[27] Julia Seiter, Oliver Amft, Mirco Rossi, and Gerhard Tröster. 2014. Discovery of activity composites using topic models: An analysis of unsupervised methods. Pervasive and Mobile Computing 15 (2014), 215-227.

[28] Janine H. Stubbe, Marleen H. M. de Moor, Dorret I. Boomsma, and Eco J. C. de Geus. 2007. The association between exercise participation and well-being: A co-twin study. Preventive Medicine 44, 2 (2007), 148-152.

[29] Sara Taylor, Natasha Jaques, Ehimwenma Nosakhare, Akane Sano, and Rosalind Picard. 2017. Personalized multitask learning for predicting tomorrow's mood, stress, and health. IEEE Transactions on Affective Computing 14, 8 (2017), 1.

[30] Rui Wang, Fanglin Chen, Zhenyu Chen, Tianxing Li, Gabriella Harari, Stefanie Tignor, Xia Zhou, Dror Ben-Zeev, and Andrew T. Campbell. 2014. StudentLife: Assessing mental health, academic performance and behavioral trends of college students using smartphones. In Proceedings of the ACM International foint Conference on Pervasive and Ubiquitous Computing. 3-14.

[31] Paul Wicks, Michael Massagli, Jeana Frost, Catherine Brownstein, Sally Okun, Timothy Vaughan, Richard Bradley, and James Heywood. 2010. Sharing health data for better outcomes on PatientsLikeMe. fournal of Medical Internet Research 12, 2 (2010), e19. DOI: https:// doi.org/10.2196/jmir.1549

[32] Simone Wilson-Salandy and Mary A. Nies. 2012. The effect of physical activity on the stress management, interpersonal relationships, and alcohol consumption of college freshmen. SAGE Open 2, 4 (2012), 1-4.

Received October 2019; accepted October 2019 